\title{
An introduction to 3D discrete magnetic Laplacians and noncommutative 3-tori
}

\author{
ERIK BÉDOS*
}

June 1998

\begin{abstract}
We initiate a study of spectral properties of $3 \mathrm{D}$ discrete magnetic Laplacians based on their relationship to noncommutative 3-tori.
\end{abstract}

\section{Introduction}

In nonrelativistic quantum mechanics, the Hamiltonian of a spinless charge carrier moving in space in the absence of an electric potential, but submitted to a uniform magnetic field $\mathbf{B}$, is usually taken to be given by

$$
H_{\mathbf{B}}=\frac{1}{2 m}\left(\mathbf{P}-\frac{q}{c} \mathbf{A}\right)^{2}
$$

where $m$ is the mass, $q$ the electric charge, $c$ the speed of light, $\mathbf{P}=\frac{h}{2 \pi i} \nabla$ ( $h$ denoting Planck's constant) and $\mathbf{A}$ is a vector potential for $\mathbf{B}$, i. e. $\nabla \times \mathbf{A}=\mathbf{B}$.

Up to unitary equivalence, the Hamitonian is independent of the choice of vector potential for $\mathbf{B}$ and one may choose e. g. $\mathbf{A}(\mathbf{x})=\frac{1}{2}(\mathbf{B} \times \mathbf{x})$. Further, it is well known that $H_{\mathbf{B}}$ is essentially self-adjoint on $C_{0}^{\infty}\left(\mathbb{R}^{3}\right)$, cf. [RS].

When the motion is restricted to a plane, only the component $B$ of $\mathbf{B}$ perpendicular to the plane produces some effect and one therefore considers without loss of generality the $2 \mathrm{D}$ Hamiltonian given by

$$
H_{B}=\frac{1}{2 m}\left(\mathbf{P}-\frac{q}{c} \mathbf{A}\right)^{2}
$$

where now $\mathbf{A}=\left(A_{1}, A_{2}\right)$ satisfies $\partial_{1} A_{2}-\partial_{2} A_{1}=B, H_{B}$ being then essentially self-adjoint on $C_{0}^{\infty}\left(\mathbb{R}^{2}\right)$.

The determination of the spectra of these Hamiltonians goes back to Landau in 1930 (see [Lan] or [LL]). The operator $H_{B}$ is in essence the Hamiltonian of an harmonic oscillator and its spectrum is given by

*partially supported by the Norwegian Research Council (NFR) 


$$
S p\left(H_{B}\right)=\left\{\left(n+\frac{1}{2}\right) \frac{|q| h}{2 \pi m c}|B| ; n=0,1,2, \ldots\right\} .
$$

To determine the spectrum of $H_{\mathbf{B}}$, one may assume, by rotating the axes if necessary, that $\mathbf{B}=(0,0, B)$. This is physically obvious and not difficult to check mathematically. But then it is easy to see that $H_{\mathbf{B}}$ is essentially given as $H_{B} \otimes 1+1 \otimes\left(-\frac{h^{2}}{4 \pi^{2} m} \frac{d^{2}}{d x^{2}}\right)$ on $C_{0}^{\infty}\left(\mathbb{R}^{2}\right) \otimes C_{0}^{\infty}(\mathbb{R})$, and one gets

$$
\begin{aligned}
S p\left(H_{\mathbf{B}}\right) & =S p\left(H_{B}\right)+[0,+\infty) \\
& =\left[\frac{|q| h}{4 \pi m c}\|\mathbf{B}\|,+\infty\right) .
\end{aligned}
$$

In the presence of an electric potential $V$, the resulting Hamiltonians are now the magnetic Schrödinger operators formally given by $H_{\mathbf{B}}+V$ in the 3D case and $H_{B}+V$ in the 2D case. The spectral properties of these operators have been investigated by many mathematicians and physicists ( see [Hun, CFKS] and references therein), the best understood case being when $\mathrm{V}$ is smooth and periodic ([HS, Sjo]). In some aspects, the theory of $\mathrm{C}^{*}$-algebras and the noncommutative geometry of A. Connes have shown to be a very useful tool in this study, as beautifully illustrated by the work of J. Bellissard on the integer quantum Hall effect ([Bel2, BES, Con2]).

To study qualitative behaviour of magnetic Schrödinger operators, discrete lattice models have also been considered (see e. g. [Bel1, Bel5]). In the case of a square lattice, the 2D discrete magnetic Laplacian analogous to $H_{B}$ (also called Harper's model) have fascinated many authors and is still not yet fully understood. We refer to [Bel4, Shu] for two recent reviews on this operator, although we shall recall some relevant facts in the second section. One should note here that many spectral properties of 2D discrete magnetic Laplacians rely on properties of the noncommutative 2-tori intrisically associated to them, and that the spectrum depends on the magnetic field in a much more sophisticated way than in the 2D continuous case, leading to Hofstadter's famous butterfly.

In the case of a 3D cubic lattice one may obviously introduce the discrete analogue of $H_{\mathbf{B}}$, as we do in the third section, and which is naturally named the $3 D$ discrete magnetic Laplacian. As we have not found any reference in the literature dealing explicitely with this model, our aim with these introductory notes is to initiate a mathematical study of the spectral properties of 3D discrete magnetic Laplacians, based on the properties of the noncommutative 3-tori which now enter the picture.

A nice introduction to noncommutative tori of arbitrary dimension may be found in [Rie4]. Due to the work of many hands, one has by now a quite detailed knowledge of the structure of noncommutative 2-tori (also called rotation algebras by some authors). Noncommutative 3-tori are also relatively well understood and, for convenience, we present in the fourth section a review of the 
results on these algebras needed later on. For completeness, we have included a classification result, which illustratres how the 3D case differs from the more simple 2D case.

In the fifth and final section, we obtain, as promised above, some spectral properties of 3D discrete magnetic Laplacians. Some of the 2D results have direct analogues, such as perfectness and symmetry around 0 , absence of point spectrum, band structure in the so-called rational case, continuity with respect to the magnetic field, continuity of the integrated density of states. Some other results, such as Aubry duality and the connection with almost Mathieu operators, must be somewhat reformulated. We also mention some results which may serve as a basis for computing effectively numerical approximations of the spectra of some of these Laplacians.

In the nonrational case, there are examples of discrete Laplacians with nonCantor spectrum (even connected spectrum), in contrast with the 2D case. In view of the description of the spectrum of $H_{\mathbf{B}}$ mentionned earlier, one possible guess is that this could always be true, i. e. 3D discrete magnetic Laplacians have never non-Cantor spectrum. However, in the discrete model, one does not have the same possibility of freely rotating the axes as in the continuous model, and it may therefore still happen that the spectrum exhibits a Cantor structure in some examples. In analogy with the 2D discrete case, a reasonable question is wether one can expect a Cantor spectrum whenever the associated noncommutative 3-tori is simple with a unique trace, but we have so far no result in this direction. Another question of interest (mentionned to us by J. Bellissard), which we don't take up in these notes, is wether the de Haas-van Alphen effect ([HS, Sjo]) can be observed in the 3D discrete model.

Our notation for noncommutative 3-tori differs slightly from the usual one. It comes out naturally from the heuristic physical motivation given in the third section and makes life easier in some aspects. Otherwise, we use standard notation and terminology from operator algebras, homomorphisms and isomorphisms between $\mathrm{C}^{*}$-algebras being always assumed to be *-preserving. Non-specialists in this field are invited to consult Fillmores pleasant guide [Fil] as a first source of information.

\section{On 2D discrete magnetic Laplacians}

We recall in this section a few facts about 2D discrete magnetic Laplacians, mainly for notational purposes. We refer to [Bel4, Shu] for much more detailed reviews.

Let $\theta \in \mathbb{R}$. The associated $2 D$ discrete magnetic Laplacian $\Delta_{\theta}$ on a square 
lattice is the bounded self-adjoint operator acting on $\ell^{2}\left(\mathbb{Z}^{2}\right)$ given by

$$
\begin{aligned}
\left(\Delta_{\theta} \xi\right)(m, n) & =e^{-i \pi \theta n} \xi(m+1, n)+e^{i \pi \theta n} \xi(m-1, n) \\
& +e^{i \pi \theta m} \xi(m, n+1)+e^{-i \pi \theta m} \xi(m, n-1) .
\end{aligned}
$$

To be more precise, one should substract four times the identity operator $I$ from $\Delta_{\theta}$ in the definition of the 2D discrete magnetic Laplacian, but we follow [Shu] here. Note that $\Delta_{\theta}-4 I$ reduces to the usual 2D discrete Laplacian on $\ell^{2}\left(\mathbb{Z}^{2}\right)$ when $\theta=0$. Up to some physical constant, $\theta$ represents the (vertical) component of a uniform magnetic field perpendicular to the (horizontal) square lattice, and the discrete analogue of $H_{B}$ is qualitatively given by $4 I-\Delta_{\theta}$. Note also that the operator $\Delta_{\theta}$ often appears in some unitary equivalent disguise.

One may obviously write

$$
\Delta_{\theta}=\left(U^{*}+U+V^{*}+V\right)
$$

where $U$ and $V$ are the unitaries on $\ell^{2}\left(\mathbb{Z}^{2}\right)$ defined by

$$
\begin{aligned}
& (U \xi)(m, n)=e^{i \pi \theta n} \xi(m-1, n), \\
& (V \xi)(m, n)=e^{-i \pi \theta m} \xi(m, n-1),
\end{aligned}
$$

and which satisfy the relation

$$
U V=e^{2 \pi i \theta} V U
$$

To include the case of a rectangular lattice one also considers Laplacians given by

$$
\Delta_{\theta, c}=\left(U^{*}+U\right)+c\left(V^{*}+V\right)
$$

where $c \in \mathbb{R}$, so $\Delta_{\theta}=\Delta_{\theta, 1}$.

Let $\mathcal{A}_{\theta}$ denote the $\mathrm{C}^{*}$-subalgebra of $\mathcal{B}\left(\ell^{2}\left(\mathbb{Z}^{2}\right)\right)$ generated by $U$ and $V$, i. e. $\mathcal{A}_{\theta}=\mathrm{C}^{*}(\mathrm{U}, \mathrm{V})$, so clearly $\Delta_{\theta, c} \in \mathcal{A}_{\theta}$ for all c. Then $\mathcal{A}_{\theta}$ may be identified as a noncommutative 2-torus (or rotation algebra), i. e. as the universal $\mathrm{C}^{*}$-algebra generated by two unitaries satisfying the relation $(*)$ above. The name rotation algebra stems from the decomposition of $\mathcal{A}_{\theta}$ as the crossed product of $C(\mathbb{T})$ by the action of $\mathbb{Z}$ induced by rotating $\mathbb{T}$ through the angle $2 \pi \theta$.

Let now $\beta, c \in \mathbb{R}$ and denote by $M_{\theta, \beta}$ the multiplication operator acting on $\ell^{2}(\mathbb{Z})$ associated to the function

$$
m \longrightarrow 2 \cos (2 \pi(m \theta+\beta)) .
$$

The almost Mathieu operator $H_{\theta, \beta, c}$ is then the almost periodic discrete Schrödinger operator acting on $\ell^{2}(\mathbb{Z})$ defined by

$$
H_{\theta, \beta, c}=\Delta+c M_{\theta, \beta}
$$


where $\Delta$ denotes the Laplacian on $\ell^{2}(\mathbb{Z})$ defined by

$$
(\Delta \psi)(m)=\psi(m+1)+\psi(m-1) .
$$

The spectrum of $\Delta_{\theta, c}$ is related to the spectrum of $H_{\theta, \beta, c}$ as follows :

$$
S p\left(\Delta_{\theta, c}\right)= \begin{cases}S p\left(H_{\theta, \beta, c}\right) & \text { if } \theta \notin \mathbb{Q}, \\ \bigcup_{\beta \in[0,1]} S p\left(H_{\theta, \beta, c}\right) & \text { if } \theta \in \mathbb{Q} .\end{cases}
$$

There exists an impressive amount of literature concerning the spectral properties of the operators defined in this section. Again, we refer to [Bel4, Shu] and the references given therein. Although there are many partial positive results in this direction, the main unsolved problem is the conjecture of M. Kac (the so-called "Ten Martini Problem") saying that $S p\left(\Delta_{\theta, c}\right)$ is Cantor set whenever $\theta \notin \mathbb{Q}$ and $c \neq 0$. A recent result which "almost" solves this problem when $c=1$ may be found in [Las] : the set $\left\{\theta \in \mathbb{R} \mid S p\left(\Delta_{\theta}\right)\right.$ is not a Cantor set $\}$ has Lebesgue measure zero. Another recent result concerning the behaviour of $\left\|\Delta_{\theta}\right\|$ as $\theta$ varies is contained in [BVZ].

\section{The 3D discrete magnetic Laplacian}

Let $\Theta=\left(\theta_{1}, \theta_{2}, \theta_{3}\right) \in \mathbb{R}^{3}$. As a convention, we think of vectors written this way as column vectors whenever this makes sense. Further, when $\mathbf{x} \in \mathbb{R}^{3}$, then the components of $\mathbf{x}$ are implicitely given as $x_{1}, x_{2}$ and $x_{3}$.

3.1 Definition.The 3D discrete magnetic Laplacian, or 3D DML, on a cubic lattice (associated to $\Theta$ ) is the operator $\Delta_{\Theta}$ defined on $\ell^{2}\left(\mathbb{Z}^{3}\right)$ by

$$
\begin{aligned}
\left(\Delta_{\Theta} \xi\right)(m, n, p) & =e^{i \pi\left(\theta_{2} p-\theta_{3} n\right)} \xi(m+1, n, p)+e^{-i \pi\left(\theta_{2} p-\theta_{3} n\right)} \xi(m-1, n, p) \\
& +e^{i \pi\left(\theta_{3} m-\theta_{1} p\right)} \xi(m, n+1, p)+e^{-i \pi\left(\theta_{3} m-\theta_{1} p\right)} \xi(m, n-1, p) \\
& +e^{i \pi\left(\theta_{1} n-\theta_{2} m\right)} \xi(m, n, p+1)+e^{-i \pi\left(\theta_{1} n-\theta_{2} m\right)} \xi(m, n, p-1) .
\end{aligned}
$$

To be more precise, one should substract six times the identity operator $I$ from $\Delta_{\Theta}$ to get the correct definition of this Laplacian, but we follow here common use as in the $2 \mathrm{D}$ case. To motivate the definition, we adapt the argument given in [Bel3], p. 38, in the 2D case. For clarity of exposition, we disregard the physical constants whenever possible. More generally, one defines magnetic Laplacians on graphs in an analogous way, see e. g. [LiLo, Sun].

We consider an electron described in a tight-binding model (Hückel's model) by a wave function $\xi$ in $\ell^{2}\left(\mathbb{Z}^{3}\right)$, submitted to a uniform magnetic field $\mathbf{B}$. Its energy operator $\mathcal{E}_{\mathbf{B}}$ may be qualitatively described, in a first approximation by 
means of nearest neighbours interaction, by changing the phase of each term in the energy operator of a free electron as follows:

$$
\begin{aligned}
\left(\mathcal{E}_{\mathbf{B}} \xi\right)(m, n, p) & =e^{i 2 \pi A_{1}(m, n, p)} \xi(m+1, n, p)+e^{-i 2 \pi A_{1}(m-1, n, p)} \xi(m-1, n, p) \\
& +e^{i \pi A_{2}(m, n, p)} \xi(m, n+1, p)+e^{-i 2 \pi A_{2}(m, n-1, p)} \xi(m, n-1, p) \\
& +e^{i 2 \pi A_{3}(m, n, p)} \xi(m, n, p+1)+e^{-i 2 \pi A_{3}(m, n, p-1)} \xi(m, n, p-1)
\end{aligned}
$$

where $A_{j}(m, n, p)$ represents the product of a physical constant $\rho$ by the line integral of a vector potential $\mathbf{A}$ for $\mathbf{B}$ along the segment between $(m, n, p)$ and $(m+1, n, p)$ for $j=1$, or $(m, n+1, p)$ for $j=2$, or $(m, n, p+1)$ for $j=3$.

Now, choosing $\mathbf{A}(\mathbf{x})=\frac{1}{2}(\mathbf{B} \times \mathbf{x})$ and setting $\Theta=\rho \mathbf{B}$ gives $\mathcal{E}_{\mathbf{B}}=\Delta_{\Theta}$ after a short computation. Thus $6 I-\Delta_{\Theta}$ may be considered qualitatively as a discrete analogue of $H_{\mathbf{B}}$. It is easy to check that choosing another vector potential for $\mathbf{B}$ just gives a unitarily equivalent operator.

3.2 As to be expected, $\Delta_{\Theta}$ may expressed in terms of the canonical generators of a noncommutative 3 -torus. To see this, we first etablish some notation.

We define $<,>_{\Theta}: \mathbb{Z}^{3} \times \mathbb{Z}^{3} \longrightarrow \mathbb{R}$ by

$$
\begin{aligned}
<\mathbf{x}, \mathbf{y}>_{\Theta} & =\Theta \cdot(\mathbf{x} \times \mathbf{y}) \\
& =\operatorname{det}([\Theta|\mathbf{x}| \mathbf{y}]),
\end{aligned}
$$

and $\quad \sigma_{\Theta}: \mathbb{Z}^{3} \times \mathbb{Z}^{3} \longrightarrow \mathbb{T}$ by

$$
\sigma_{\Theta}(\mathbf{x}, \mathbf{y})=e^{i \pi<\mathbf{x}, \mathbf{y}>_{\Theta}} .
$$

An elementary verification gives that $\sigma_{\Theta}$ is a normalized 2-cocycle on $\mathbb{Z}^{3}$ with values in the circle group $\mathbb{T}$ as defined in $[\mathrm{ZM}]$ (see also [Bac, BB]) and we may form the associated projective regular representation $\lambda_{\Theta}$ of $\mathbb{Z}^{3}$ on $\ell^{2}\left(\mathbb{Z}^{3}\right)$, which is defined by

$$
\left(\lambda_{\Theta}(\mathbf{y}) \xi\right)(\mathbf{x})=\sigma_{\Theta}(-\mathbf{x}, \mathbf{y}) \xi(\mathbf{x}-\mathbf{y})
$$

and satisfies

$$
\begin{aligned}
\lambda_{\Theta}(\mathbf{x}) \lambda_{\Theta}(\mathbf{y}) & =\sigma_{\Theta}(\mathbf{x}, \mathbf{y}) \lambda_{\Theta}(\mathbf{x}+\mathbf{y}) \\
& =e^{i 2 \pi<\mathbf{x}, \mathbf{y}>_{\Theta}} \lambda_{\Theta}(\mathbf{y}) \lambda_{\Theta}(\mathbf{x})
\end{aligned}
$$

for all $\mathbf{x}, \mathbf{y} \in \mathbb{Z}^{3}$.

The operators $\lambda_{\Theta}(\mathbf{y})$ are called magnetic translation operators in [Bro] (see also $[\mathrm{Zak}])$. 
Denoting by $\mathbf{e}_{1}, \mathbf{e}_{2}$ and $\mathbf{e}_{3}$ the canonical generators of $\mathbb{Z}^{3}$ and setting

$$
U_{j}=\lambda_{\Theta}\left(\mathbf{e}_{j}\right), j=1,2,3,
$$

we then get the following relations between the unitaries $U_{1}, U_{2}$ and $U_{3}$

$$
\left\{\begin{array}{l}
U_{1} U_{2}=e^{i 2 \pi \theta_{3}} U_{2} U_{1}, \\
U_{2} U_{3}=e^{i 2 \pi \theta_{1}} U_{3} U_{2}, \\
U_{3} U_{1}=e^{i 2 \pi \theta_{2}} U_{1} U_{3} .
\end{array}\right.
$$

It is a simple exercise to check that

$$
\lambda_{\Theta}(\mathbf{y})=e^{-i \pi\left(\theta_{1} y_{2} y_{3}-\theta_{2} y_{1} y_{3}+\theta_{3} y_{1} y_{2}\right)} U_{1}^{y_{1}} U_{2}^{y_{2}} U_{3}^{y_{3}}
$$

and that

$$
\Delta_{\Theta}=U_{1}^{*}+U_{1}+U_{2}^{*}+U_{2}+U_{3}^{*}+U_{3},
$$

so $\Delta_{\Theta}$ is bounded, self-adjoint and has norm less or equal to 6 . To include the case of a box lattice, not necessarily cubic, we also consider 3D discrete magnetic Laplacians $\Delta_{\Theta, c}\left(\mathbf{c} \in \mathbb{R}^{3}\right)$ defined by

$$
\Delta_{\Theta, \mathrm{c}}=c_{1}\left(U_{1}^{*}+U_{1}\right)+c_{2}\left(U_{2}^{*}+U_{2}\right)+c_{3}\left(U_{3}^{*}+U_{3}\right),
$$

so $\Delta_{\Theta}=\Delta_{\Theta,(1,1,1)}$.

3.3 Definition. We define the noncommutative 3-torus (associated to $\Theta$ ) as the $\mathrm{C}^{*}$-subalgebra $\mathcal{A}_{\Theta}$ of $\mathcal{B}\left(\ell^{2}\left(\mathbb{Z}^{3}\right)\right)$ generated by $U_{1}, U_{2}$ and $U_{3}$.

Obviously, we have $\Delta_{\Theta, \mathbf{c}} \in \mathcal{A}_{\Theta}$.

Let $C_{r}^{*}\left(\mathbb{Z}^{3}, \sigma_{\Theta}\right)$ denote the reduced twisted group $C^{*}$-algegra of $\mathbb{Z}^{3}$ associated with $\sigma_{\Theta}($ see $[\mathrm{ZM}])$, i. e. the $\mathrm{C}^{*}$-subalgebra of $\mathcal{B}\left(\ell^{2}\left(\mathbb{Z}^{3}\right)\right)$ generated by the set $\left\{\lambda_{\Theta}(\mathbf{y}) \mid \mathbf{y} \in \mathbb{Z}^{3}\right\}$. Due to (2), it is clear that

$$
\mathcal{A}_{\Theta}=C_{r}^{*}\left(\mathbb{Z}^{3}, \sigma_{\Theta}\right) .
$$

Further, it is well-known that

$\mathcal{A}_{\Theta}$ is the universal $\mathrm{C}^{*}$-algebra generated by three unitaries $U_{1}, U_{2}$ and $U_{3}$ satisfying (1).

This result is a part of the folklore on this subject. For completeness, let us briefly sketch one possible way to prove it. As $\mathbb{Z}^{3}$ is an amenable group, one knows that $C_{r}^{*}\left(\mathbb{Z}^{3}, \sigma_{\Theta}\right)$ is canonically isomorphic to the full twisted group $\mathrm{C}^{*}$ algebra $C^{*}\left(\mathbb{Z}^{3}, \sigma_{\Theta}\right)$ (cf. [ZM]). Then the result follows easily by exploiting the universal properties of $C^{*}\left(\mathbb{Z}^{3}, \sigma_{\Theta}\right)$ and the fact that whenever three unitaries $U_{1}, U_{2}$ and $U_{3}$ satisfy (1), then one may define unitaries $W(\mathbf{y})$ for $\mathbf{y} \in \mathbb{Z}^{3}$ by

$$
W(\mathbf{y})=e^{-i \pi\left(\theta_{1} y_{2} y_{3}-\theta_{2} y_{1} y_{3}+\theta_{3} y_{1} y_{2}\right)} U_{1}^{y_{1}} U_{2}^{y_{2}} U_{3}^{y_{3}},
$$


which then satisfy $W(\mathbf{x}) W(\mathbf{y})=\sigma_{\Theta}(\mathbf{x}, \mathbf{y}) W(\mathbf{x}+\mathbf{y})$ for all $\mathbf{x}, \mathbf{y} \in \mathbb{Z}^{3}$.

From this universal property of noncommutative 3-tori, it is clear that, up to isomorphism, $\mathcal{A}_{\Theta}$ is only determined up to an even permutation of the components of $\Theta$. It is a nontrivial fact that this is also true up to a general permutation, as we shall see in 4.8 .

3.4 To give a small appetizer of how noncommutative 3-tori are useful when studying spectral properties of 3D DML's, let us show that the spectrum of $\Delta_{\Theta}$ may not be a Cantor set even when $\Theta$ has some nonrational components.

Let $\theta \in \mathbb{R}$ and set $\Theta=(0,-\theta, \theta)$. Let $U$ and $V$ denote the canonical generators of $\mathcal{A}_{\theta}$ and set $W=-V$. Then $U, V$ and $W$ are unitaries satisfying $U V=e^{i 2 \pi \theta} V U, V W=W V, W U=e^{-i 2 \pi \theta} U W$. Hence, by the universal proprties of $\mathcal{A}_{\Theta}$, there exists an homomorphism $\Pi: \mathcal{A}_{\Theta} \longrightarrow \mathcal{A}_{\theta}$ such that $\Pi\left(U_{1}\right)=U, \Pi\left(U_{2}\right)=V$ and $\Pi\left(U_{3}\right)=W$. But then we have $\Pi\left(\Delta_{\Theta}\right)=U^{*}+U$. As it is well-known that $S p\left(U^{*}+U\right)=[-2,2]$, this implies that $[-2,2] \subseteq S p\left(\Delta_{\Theta}\right)$. So $S p\left(\Delta_{\Theta}\right)$ is not a Cantor set for this choice of $\Theta$ (even when $\theta \notin \mathbb{Q}$ ).

3.5 We conclude this section with some remarks on noncommutative $n$-tori and the different uses of notation in the literature.

To each skew-symmetric matrix $R=\left[r_{j k}\right] \in M_{n}(\mathbb{R}), n \geq 2$, one may associate a noncommutative $n$-torus $\mathcal{A}_{R}$ as the universal $\mathrm{C}^{*}$-algebra generated by $n$ unitaries $U_{1}, U_{2}, \ldots, U_{n}$ satisfying

$$
U_{j} U_{k}=e^{i 2 \pi r_{j k}} U_{k} U_{j}, 1 \leq j, k \leq n,
$$

which may be taken to be defined as the twisted group C*-algebra $C_{r}^{*}\left(\mathbb{Z}^{n}, \sigma_{R}\right)$ $\left(\simeq C^{*}\left(\mathbb{Z}^{n}, \sigma_{R}\right)\right.$ since $\mathbb{Z}^{n}$ is amenable), where $\sigma_{R}$ is the 2-cocycle on $\mathbb{Z}^{n}$ with values in $\mathbb{T}$ defined by

$$
\sigma_{R}(\mathbf{x}, \mathbf{y})=e^{i \pi\left(\mathbf{x}^{t} R \mathbf{y}\right)}
$$

This was apparently first observed by Elliott in [Ell2]. We caution that he and many other authors use the transpose of $R$ instead of $R$ in (3), which is only a matter a convenience, but may cause that one comes out with a sign off if one is not cautious, as pointed out in [Rie2].

Some authors prefer to regard $\mathcal{A}_{R}$ as associated to the antisymmetric bicharacter on $\mathbb{Z}^{n}$ (or, equivalently, the character on the second exterior power $\mathbb{Z}^{n} \wedge \mathbb{Z}^{n}$ ) given by

$$
\rho_{R}(\mathbf{x}, \mathbf{y})=\sigma_{R}(\mathbf{x}, \mathbf{y}) \overline{\sigma_{R}(\mathbf{y}, \mathbf{x})}=e^{i 2 \pi\left(\mathbf{x}^{t} R \mathbf{y}\right)} .
$$

Beware that this doesn't mean that they take $\mathcal{A}_{R}$ to be defined as $C_{r}^{*}\left(\mathbb{Z}^{n}, \rho_{R}\right)$.

There is a natural notion of cohomological equivalence between normalized 2-cocycles on the same group (see e. g. [ZM, Bac, BB, OPT]) with the property 
that cohomologous 2-cocycles give rise to spatially isomorphic reduced (resp. full) twisted group $\mathrm{C}^{*}$-algebras. (This reflects in fact the freeness of choosing different vector potentials for the same magnetic field in the case of $\mathbb{Z}^{2}$ or $\mathbb{Z}^{3}$ ). Up to cohomological equivalence, all normalized 2-cocycles on $\mathbb{Z}^{n}$ are given as some $\sigma_{R}, R$ skew-symmetric in $M_{n}(\mathbb{R})$, cf. [Bac, BB]. For example, the 2-cocycle $\rho_{M}(\mathbf{x}, \mathbf{y})=e^{i 2 \pi\left(\mathbf{x}^{t} M \mathbf{y}\right)}, M \in M_{n}(\mathbb{R})$, is cohomologous to $\sigma_{R}$ with $R=M-M^{t}$.

For a given $\Theta \in \mathbb{R}^{3}$, our $\mathcal{A}_{\Theta}$ corresponds to $\mathcal{A}_{R}$ where

$$
R=\left[\begin{array}{ccc}
0 & \theta_{3} & -\theta_{2} \\
-\theta_{3} & 0 & \theta_{1} \\
\theta_{2} & -\theta_{1} & 0
\end{array}\right]
$$

If we had chosen the vector potential $\tilde{\mathbf{A}}(\mathbf{x})=\left(b_{2} x_{3}, b_{3} x_{1}, b_{1} x_{2}\right)$ for $\mathbf{B}$ instead of $\mathbf{A}$ in 3.1 , this would have led us to the 2 -cocycle $\rho_{M}$ on $\mathbb{Z}^{3}$, where

$$
M=\left[\begin{array}{ccc}
0 & \theta_{3} & 0 \\
0 & 0 & \theta_{1} \\
\theta_{2} & 0 & 0
\end{array}\right],
$$

which is cohomologous to $\sigma_{R}$ with $R$ as above .

As will be seen in the next sections, our "vector" notation for 3-tori has some advantages.

Finally, it should by now be obvious that one may also consider $n \mathrm{D}$ discrete magnetic Laplacians belonging to noncommutative $n$-tori, but we shall stick to the 3D case in this paper, even if some of the results are also valid in higher dimensions.

\section{A review of some properties of noncommuta- tive 3-tori}

We use freely the notation introduced in the previous sections. Let $\Theta \in \mathbb{R}^{3}$. We denote by $U_{1}, U_{2}$ and $U_{3}$ the canonical generators of $\mathcal{A}_{\Theta}$, and by $\mathcal{W}_{\Theta}$ the twisted group von Neumann algebra $v N\left(\mathbb{Z}^{3}, \sigma_{\Theta}\right)$, i. e. the weak closure of $\mathcal{A}_{\Theta}=C_{r}^{*}\left(\mathbb{Z}^{3}, \sigma_{\Theta}\right)$ in $\mathcal{B}\left(\ell^{2}\left(\mathbb{Z}^{3}\right)\right)$.

4.1 Let $\tilde{\Theta} \in \mathbb{R}^{3}, \tilde{\Theta}=\Theta\left(\bmod \mathbb{Z}^{3}\right)$. It is quite (but not totally) obvious that $\mathcal{A}_{\tilde{\Theta}}$ and $\mathcal{A}_{\Theta}\left(\right.$ resp. $\mathcal{W}_{\tilde{\Theta}}$ and $\left.\mathcal{W}_{\Theta}\right)$ are isomorphic. Anyhow, it follows from the universal property of noncommutative 3 -tori that there exists an isomorphism $\pi$ from $\mathcal{A}_{\tilde{\Theta}}$ onto $\mathcal{A}_{\Theta}$ satisfying

$$
\pi\left(\tilde{U}_{j}\right)=U_{j}, j=1,2,3
$$


where $\tilde{U}_{1}, \bar{U}_{2}, \tilde{U}_{3}$ denote the canonical generators of $\mathcal{A}_{\tilde{\Theta}}$.

4.2 For $\mathbf{x} \in \mathbb{Z}^{3}$, let $\delta_{\mathbf{x}} \in \ell^{2}\left(\mathbb{Z}^{3}\right)$ be the delta function at $\mathbf{x}$ and set $\delta=\delta_{\mathbf{0}}$. Then we have

$$
\lambda_{\Theta}(\mathbf{y}) \delta_{\mathbf{x}}=\sigma_{\Theta}(\mathbf{y}, \mathbf{x}) \delta_{\mathbf{y}+\mathbf{x}}
$$

and especially $\lambda_{\Theta}(\mathbf{y}) \delta=\delta_{\mathbf{y}}$, for all $\mathbf{x}, \mathbf{y} \in \mathbb{Z}^{3}$.

It is well-known (cf. [ZM]), and easy to check using (4), that the vector $\delta$ is cyclic, tracial and separating for $\mathcal{W}_{\Theta}\left(\right.$ and $\left.\mathcal{A}_{\Theta}\right)$. Hence the map $\tau: \mathcal{W}_{\Theta} \longrightarrow \mathbb{C}$ defined by $\tau(W)=\left\langle W \delta, \delta>\right.$ is a faithful normal tracial state on $\mathcal{W}_{\Theta}$ which satisfies (and is uniquely determined by)

$$
\tau\left(\lambda_{\Theta}(\mathbf{y})\right)= \begin{cases}1 & \mathbf{x}=\mathbf{0} \\ 0 & \mathbf{x} \neq \mathbf{0}\end{cases}
$$

We also denote by $\tau$ the restriction of $\tau$ to $\mathcal{A}_{\Theta}$. The trace $\tau$ is the analogue of integration with repect to normalized Haar measure on $\mathbb{T}^{3}$. The algebra $\mathcal{A}_{\Theta}$ (resp. $\mathcal{W}_{\Theta}$ ) is a stably finite $\mathrm{C}^{*}$-algebra (resp. a finite von Neumann algebra), which is isomorphic to $C\left(\mathbb{T}^{3}\right)$ (resp. $L^{\infty}\left(\mathbb{T}^{3}\right)$ ) via Fourier transform when $\Theta=0$ $\left(\bmod \mathbb{Z}^{3}\right)$. It is not difficult to show (e. g. by "pre" Tomita-Takesaki theory) that the commutant of $\mathcal{W}_{\Theta}$ is $\mathcal{W}_{(-\Theta)}$. This supports the idea that elements of $\mathcal{W}_{\Theta}$ may be thought of as generalized periodic discrete Schrödinger operators as they commute with the magnetic translation operators associated to $(-\Theta)$.

4.3 One of the main tool for doing analysis in $\mathcal{W}_{\Theta}$ is Fourier analysis.

For each $W \in \mathcal{W}_{\Theta}$ we define its Fourier transform $\widehat{W} \in \ell^{2}\left(\mathbb{Z}^{3}\right)$ by $\widehat{W}=W \delta$.

Then we have

$$
\widehat{W}(\mathbf{x})=<W \delta, \delta_{\mathbf{x}}>=\tau\left(W \lambda_{\Theta}(\mathbf{x})^{*}\right), \mathbf{x} \in \mathbb{Z}^{3} .
$$

If we denote by $\|\cdot\|_{2}$ the norm on $\mathcal{W}_{\Theta}$ defined by $\|W\|_{2}^{2}=\tau\left(W^{*} W\right)=\|\widehat{W}\|_{2}^{2}$, then the Fourier series of $\mathrm{W}$, defined as

$$
\sum_{\mathbf{x} \in \mathbb{Z}^{3}} \widehat{W}(\mathbf{x}) \lambda_{\Theta}(\mathbf{x})
$$

converges to $\mathrm{W}$ in $\|\cdot\|_{2}$-norm, and $\mathrm{W}$ is therefore uniquely determined by $\widehat{W}$ as an element of $\mathcal{W}_{\Theta}$. As usual, the Fourier series of $W \in \mathcal{W}_{\Theta}$ doesn't necessarily converges to $W$ in operator norm, even if $W \in \mathcal{A}_{\Theta}$. But it does if $W$ belongs to the smooth canonical subalgebra $\mathcal{A}_{\Theta}^{\infty}:=\left\{A \in \mathcal{A}_{\Theta} \mid \widehat{A} \in S\left(\mathbb{Z}^{3}\right)\right\}$ of $\mathcal{A}_{\Theta}$, where $S\left(\mathbb{Z}^{3}\right)\left(\simeq C^{\infty}\left(\mathbb{T}^{3}\right)\right)$ denotes the Schwartz space of rapidly decreasing complex functions on $\mathbb{Z}^{3}$.

4.4 There exists a canonical dual ergodic action $\alpha$ of $\mathbb{T}^{3} \simeq \widehat{\mathbb{Z}^{3}}$ on $\mathcal{W}_{\Theta}$ (cf. $[\mathrm{OPT}])$ satisfying

$$
\alpha_{\gamma}\left(U_{j}\right)=\gamma_{j} U_{j}, \gamma \in \mathbb{T}^{3}, j=1,2,3
$$


Each $\alpha_{\gamma}$ is in fact implemented by the unitary $U_{\gamma}$ on $\ell^{2}\left(\mathbb{Z}^{3}\right)$ defined by $U_{\gamma} \xi(\mathbf{x})=\gamma(\mathbf{x}) \xi(\mathbf{x})$, and we have $\widehat{\alpha_{\gamma}(W)}=\gamma \widehat{W}$ for all $\gamma \in \mathbb{T}^{3}, W \in \mathcal{W}_{\Theta}$, from which the ergodicity of $\alpha$ easily follows. The action $\alpha$ obviously restricts to an action on $\mathcal{A}_{\Theta}$, which we also denote by $\alpha$. It is not difficult to check that the space of $C^{\infty}$-vectors for $\alpha$ is just $\mathcal{A}_{\Theta}^{\infty}$. For each $k=1,2,3$, one may associate the infinitesimal generator $\partial_{k}$ for this action in the $k$-th direction of $\mathbb{T}^{3}$, which is then a *-derivation on $\mathcal{A}_{\Theta}^{\infty}$ satisfying

$$
\partial_{k}(A)=\sum_{\mathbf{x} \in \mathbb{Z}^{3}} 2 \pi i x_{k} \widehat{A}(\mathbf{x}) \lambda_{\Theta}(\mathbf{x}) .
$$

4.5 We denote by $C_{\Theta}$ the subgroup of $\mathbb{Z}^{3}$ given by

$$
\begin{aligned}
C_{\Theta} & =\left\{\mathbf{x} \in \mathbb{Z}^{3} \mid<\mathbf{x}, \mathbf{y}>_{\Theta} \in \mathbb{Z} \text { for all } \mathbf{y} \in \mathbb{Z}^{3}\right\} \\
& =\left\{\mathbf{x} \in \mathbb{Z}^{3} \mid \mathbf{x} \times \Theta \in \mathbb{Z}^{3}\right\} .
\end{aligned}
$$

A quick application of Fourier analysis gives that the centre of $\mathcal{W}_{\Theta}$ is the von Neumann subalgebra of $\mathcal{W}_{\Theta}$ generated by $\left\{\lambda_{\Theta}(\mathbf{x}) \mid \mathbf{x} \in C_{\Theta}\right\}$. It is then not very difficult to conclude that the centre of $\mathcal{A}_{\Theta}$ is the $\mathrm{C}^{*}$-algebra generated by $\left\{\lambda_{\Theta}(\mathbf{x}) \mid \mathbf{x} \in C_{\Theta}\right\}$ (see also [Ell2], p.170-171).

Let $d(\Theta)$ denote the dimension of $\mathbb{Q}+\theta_{1} \mathbb{Q}+\theta_{2} \mathbb{Q}+\theta_{3} \mathbb{Q}$ as a vector space over $\mathbb{Q}$, so $d(\Theta) \in\{1,2,3,4\}$. We say that $\Theta$ (or $\mathcal{A}_{\Theta}$ ) is rational whenever $d(\Theta)=1$, i. e. $\Theta$ has only rational components, while we say that $\Theta$ (or $\mathcal{A}_{\Theta}$ ) is totally irrational whenever $d(\Theta)=4$, i. e. $1, \theta_{1}, \theta_{2}$ and $\theta_{3}$ are rationally independent .

It is a simple exercise to verify that $C_{\Theta}=\{0\}$ if and only if $d(\Theta) \in\{3,4\}$. This situation is often refered to as the nondegenerate case in the literature.

The nontrivial implications in the following theorem are special cases of results in [Sla], see also [Pac].

Theorem. The following statements are equivalent :

(i) $d(\Theta) \in\{3,4\}$

(ii) $\mathcal{A}_{\Theta}$ is simple

(iii) $\mathcal{A}_{\Theta}$ has a unique trace

(iv) $\mathcal{W}_{\Theta}$ is a factor

(v) $\mathcal{A}_{\Theta}$ has a trivial centre

As $\mathbb{Z}^{3}$ is amenable, it follows from [Con1] that $\mathcal{W}_{\Theta}$ is always injective, and that $\mathcal{W}_{\Theta}$ is isomorphic to the hyperfinite $I I_{1}$-factor whenever $d(\Theta) \in\{3,4\}$.

Concerning the structure of noncommutative 3-tori, we shall see in 4.9 that rational 3-tori are subhomogeneous. More generally, it has recently been shown in [Lin], building heavily on [EE] and [EL1, EL2], that any noncommutative 3 -torus is the inductive limit of type $\mathrm{I} \mathrm{C}^{*}$-algebras (see [Lin],Corollary 1 , for a more precise statement; this result holds for almost all noncommutative $n$ tori, as shown in [Boc]). This strengthens the fact known earlier that $\mathcal{A}_{\Theta}$ is 
nuclear. It is also known that every nonrational noncommutative torus is socalled approximately divisible and therefore has stable rank 1 and real rank zero whenever simple (cf. [BKR]).

4.6 We shall need some results concerning the K-theory of noncommutative 3 -tori. Short overviews of $\mathrm{K}$-theory for $\mathrm{C}^{*}$-algebras may be found in [Bel1, Bel5, Fil]. A much more comprehensive treatise is [Bla], which includes a proof of the Pimsner-Voiculescu (PV-)sequence for crossed products of $\mathrm{C}^{*}$-algebras by actions of $\mathbb{Z}$. One of the main motivation in the original paper of Pimsner and Voiculescu ([PV]) was that this sequence enabled them to compute the K-theory of $\mathcal{A}_{\theta}$. They obtained

$$
K_{0}\left(\mathcal{A}_{\theta}\right) \simeq \mathbb{Z}^{2} \simeq K_{1}\left(\mathcal{A}_{\theta}\right) .
$$

By taking into account the fundamental construction of Rieffel ([Rie1]) of a nontrivial projection in $\mathcal{A}_{\theta}$ of trace $\theta$ when $\theta \in(0,1)$, they showed further that $\mathcal{A}_{\theta}$ is isomorphic to $\mathcal{A}_{\tilde{\theta}}$ if and only if $\theta=\tilde{\theta}$ or $1-\tilde{\theta}(\bmod \mathbb{Z})$.

Now one may decompose a noncommutative 3 -torus $\mathcal{A}_{\Theta}$ as the crossed product of $\mathcal{A}_{\theta_{3}}$ by an action of $\mathbb{Z}$ as follows. Using 4.4, let $\alpha_{1}$ denote the automorphism of $\mathcal{A}_{\theta_{3}}$ satisfying $\alpha_{1}(U)=e^{i 2 \pi \theta_{2}} U, \alpha_{1}(V)=e^{-i 2 \pi \theta_{1}} V$, where $U$ and $V$ denote the canonical generators of $\mathcal{A}_{\theta_{3}}$. This automorphism induces an action of $\mathbb{Z}$ on $\mathcal{A}_{\theta_{3}}$ and the resulting $\mathrm{C}^{*}$-crossed product is then isomorphic to $\mathcal{A}_{\Theta}$, since it is generated by the unitaries $U, V$ and $W$, where $W$ is the unitary inside the crossed product which implements $\alpha_{1}$, and satisfies the same universal property as $\mathcal{A}_{\Theta}$, as follows readily from the universal property of the crossed product.

By exploiting the PV-sequence associated to this decomposition, one gets

$$
K_{0}\left(\mathcal{A}_{\Theta}\right) \simeq \mathbb{Z}^{4} \simeq K_{1}\left(\mathcal{A}_{\Theta}\right) .
$$

This result is due to Elliott ([El12], see also [Rie1]) in the more general case of noncommutative $n$-tori, using an analogous inductive step. When choosing $n=3$, the main result of [El12] says that if one denotes by $\tau_{*}$ the canonical homomorphism from $K_{0}\left(\mathcal{A}_{\Theta}\right)$ into $\mathbb{R}$ induced by the tracial state $\tau$ on $\mathcal{A}_{\Theta}$ defined in 4.2 , then one has

$$
\tau_{*}\left(K_{0}\left(\mathcal{A}_{\Theta}\right)\right)=\mathbb{Z}+\theta_{1} \mathbb{Z}+\theta_{2} \mathbb{Z}+\theta_{3} \mathbb{Z}
$$

and also $\tau_{*}=\tau_{*}^{\prime}$ whenever $\tau^{\prime}$ is another tracial state on $\mathcal{A}_{\Theta}$ (if in the degenerate case).

4.7 One may also decompose $\mathcal{A}_{\Theta}$ as a twisted $\mathrm{C}^{*}$-crossed product ([ZM]) of $C(\mathbb{T})$ by a twisted action $(\beta, \omega)$ of $\mathbb{Z}^{2}$ as follows.

We define $\beta: \mathbb{Z}^{2} \longrightarrow \operatorname{Aut}(C(\mathbb{T}))$ by

$$
\left(\beta_{\mathbf{m}}(f)\right)(z)=f\left(e^{-i 2 \pi\left(m_{1} \theta_{2}-m_{2} \theta_{1}\right)} z\right)
$$


and $\omega: \mathbb{Z}^{2} \times \mathbb{Z}^{2} \longrightarrow \mathbb{T}$ by

$$
\omega(\mathbf{m}, \mathbf{n})=e^{i \pi \theta_{3}\left(\left(m_{1} n_{2}-m_{2} n_{1}\right)\right.} .
$$

It is not difficult to verify that this gives a twisted action of $\mathbb{Z}^{2}$ on $C(\mathbb{T})$ and that the resulting twisted $\mathrm{C}^{*}$-crossed product is isomorphic to $\mathcal{A}_{\Theta}$, using the universal defining properties of both these $\mathrm{C}^{*}$-algebras. As we will not use this result explicitely later on, we don't elaborate on the proof. We mention it because it is related to the connection between 3D discrete magnetic Laplacians and 2D almost Mathieu operators we shall etablish in 5.10. One may also use it in conjunction with the main theorem in [Bed1] to obtain another proof of Slawny's result recalled in 4.5 .

4.8 We will now present a classification theorem for noncommutative 3-tori, which illustrates that not all results concerning noncommutative 2-tori do have a direct analogue in 3D. Up to the result mentioned in (d) below, this theorem is due to Brenken ([Bre]). Our presentation will be somewhat different from his, and hopefully more straightforward.

Let us first remark that the canonical action of $S L(2, \mathbb{Z})$ on $C\left(\mathbb{T}^{2}\right)$ as toral automorphisms may be generalized to produce an action of $S L(2, \mathbb{Z})$ on any noncommutative 2-torus ([Wat]). If one carries out the same idea in 3D, then the outcome may be described as follows:

(a) Let $A \in S L(3, \mathbb{Z})$. Then there exists an isomorphism $\iota_{A}$ from $\mathcal{A}_{\Theta}$ onto $\mathcal{A}_{A \Theta}$ such that $\iota_{A}\left(\lambda_{\Theta}(\mathbf{y})\right)=\lambda_{A \Theta}(A \mathbf{y})$ for all $\mathbf{y} \in \mathbb{Z}^{3}$.

Sketch of the proof. The clue is that we have

$$
\begin{aligned}
<A \mathbf{x}, A \mathbf{y}>_{A \Theta} & =\operatorname{det}([A \Theta|A \mathbf{x}| A \mathbf{y}]) \\
& =\operatorname{det}(A[\Theta|\mathbf{x}| \mathbf{y}])=\operatorname{det}(A) \operatorname{det}([\Theta|\mathbf{x}| \mathbf{y}]) \\
& =<\mathbf{x}, \mathbf{y}>_{\Theta} \text { for all } \mathbf{x}, \mathbf{y} \in \mathbb{Z}^{3}
\end{aligned}
$$

Now define $U_{A}$ as the unitary on $\ell^{2}\left(\mathbb{Z}^{3}\right)$ given by

$$
\left(U_{A} \xi\right)(\mathbf{x})=\xi\left(A^{-1} \mathbf{x}\right) .
$$

Using the clue observation above, a short computation gives

$$
U_{A} \lambda_{\Theta}(\mathbf{y}) U_{A}^{*}=\lambda_{A \Theta}(A \mathbf{y})
$$

for all $\mathrm{y} \in \mathbb{Z}^{3}$, and it is then clear that the restriction of $\operatorname{Ad}\left(U_{A}\right)$ to $\mathcal{A}_{\Theta}$ has the desired properties of $\iota_{A}$.

We remark that if one starts with $B \in G L(3, \mathbb{Z})$ and proceeds analogously, the outcome is then just $\iota_{A}$, where $A=-B \in S L(3, \mathbb{Z})$. 
Obviously, we may introduce an equivalence relation $\sim$ on $\mathbb{R}^{3}$ by setting

$$
\Theta \sim \tilde{\Theta} \Longleftrightarrow \text { there exists } A \in S L(3, \mathbb{Z}) \text { such that } \tilde{\Theta}=A \Theta\left(\bmod \mathbb{Z}^{3}\right) .
$$

By combining 4.1 and (a) above we get :

$$
\Theta \sim \tilde{\Theta} \Longrightarrow \mathcal{A}_{\Theta} \simeq \mathcal{A}_{\tilde{\Theta}}
$$

We may also introduce another equivalence relation $\approx$ on $\mathbb{R}^{3}$ by setting

$$
\Theta \approx \tilde{\Theta} \Longleftrightarrow \text { there exists } B \in G L(3, \mathbb{Z}) \text { such that } \tilde{\Theta}=B \Theta\left(\bmod \mathbb{Z}^{3}\right) \text {. }
$$

Then we have

$$
\mathcal{A}_{\Theta} \simeq \mathcal{A}_{\tilde{\Theta}} \Longrightarrow \Theta \approx \tilde{\Theta} .
$$

Sketch of the proof. We adapt the line of proof given by Yin ([Yin]) in the $2 \mathrm{D}$ case. Assume that $\phi$ is an isomorphism from $\mathcal{A}_{\Theta}$ onto $\mathcal{A}_{\tilde{\Theta}}$. Using 4.1, we may assume that $0<\theta_{j}, \tilde{\theta}_{j} \leq 1, j=1,2,3$. We denote by $\tilde{\tau}$ the canonical trace on $\mathcal{A}_{\tilde{\Theta}}$.

Using the PV-sequence on the decomposition of a noncommutative 3-tori as a crossed product of a noncommutative 2 -tori by an action of $\mathbb{Z}$ described in 4.6, together with the appendix of [PV], it is not difficult to see that there exist (Rieffel or Bott) projections $p_{1}, p_{2}, p_{3}$ (resp. $\left.\tilde{p}_{1}, \tilde{p}_{2}, \tilde{p}_{3}\right)$ in $\mathcal{A}_{\theta}$ or in $M_{2}\left(\mathcal{A}_{\Theta}\right)$ (resp. in $\mathcal{A}_{\tilde{\Theta}}$ or in $M_{2}\left(\mathcal{A}_{\tilde{\Theta}}\right)$ ) such that

$$
\begin{aligned}
& K_{0}\left(\mathcal{A}_{\Theta}\right) \simeq \mathbb{Z}^{4} \text { with generators }[1],\left[p_{1}\right],\left[p_{2}\right],\left[p_{3}\right], \\
& K_{0}\left(\mathcal{A}_{\tilde{\Theta}}\right) \simeq \mathbb{Z}^{4} \text { with generators }[1],\left[\tilde{p}_{1}\right],\left[\tilde{p}_{2}\right],\left[\tilde{p}_{3}\right],
\end{aligned}
$$

and

$$
\tau_{*}\left(\left[p_{k}\right]\right)=\theta_{k}, \tilde{\tau}_{*}\left(\left[\tilde{p}_{k}\right]\right)=\tilde{\theta}_{k}, k=1,2,3 .
$$

Let $\phi_{*}$ denote the isomorphism from $K_{0}\left(\mathcal{A}_{\Theta}\right) \simeq[1] \mathbb{Z} \oplus\left[p_{1}\right] \mathbb{Z} \oplus\left[p_{2}\right] \mathbb{Z} \oplus\left[p_{3}\right] \mathbb{Z}$ onto $K_{0}\left(\mathcal{A}_{\tilde{\Theta}}\right) \simeq[1] \mathbb{Z} \oplus\left[\tilde{p}_{1}\right] \mathbb{Z} \oplus\left[\tilde{p}_{2}\right] \mathbb{Z} \oplus\left[\tilde{p}_{3}\right] \mathbb{Z}$ induced by $\phi$. As $\phi_{*}([1])=[1]$, the matrix of $\phi_{*}$ with respect to these decompositions must be of the form

$$
\phi_{*}=\left[\begin{array}{cccc}
1 & a_{1} & a_{2} & a_{3} \\
0 & b_{11} & b_{12} & b_{13} \\
0 & b_{21} & b_{22} & b_{23} \\
0 & b_{31} & b_{32} & b_{33}
\end{array}\right],
$$

where $a_{1}, a_{2}, a_{3} \in \mathbb{Z}$ and $B=\left[b_{j k}\right] \in G L(3, \mathbb{Z})$. 
Now, as $\tilde{\tau} \circ \phi$ is also a tracial state on $\mathcal{A}_{\Theta}$, we know from [Ell2], cf. 4.6, that $\tau_{*}=(\tilde{\tau} \circ \phi)_{*}=\tilde{\tau}_{*} \circ \phi_{*}$. Hence, for each $k=1,2,3$, we get

$$
\begin{aligned}
\theta_{k} & =\tau_{*}\left(\left[p_{k}\right]\right)=\tilde{\tau}_{*}\left(\phi_{*}\left(\left[p_{k}\right]\right)\right. \\
& =\tilde{\tau}_{*}\left(a_{k}[1]+b_{1 k}\left[\tilde{p}_{1}\right]+b_{2 k}\left[\tilde{p}_{2}\right]+b_{3 k}\left[\tilde{p}_{3}\right]\right) \\
& =a_{k}+b_{1 k} \tilde{\theta}_{1}+b_{2 k} \tilde{\theta}_{2}+b_{3 k} \tilde{\theta}_{3} .
\end{aligned}
$$

This gives $\Theta=B^{t} \tilde{\Theta}\left(\bmod \mathbb{Z}^{3}\right)$ and therefore $\Theta \approx \tilde{\Theta}$ as desired.

The next statement looks quite innocent at first sight, but is in fact non trivial, as it relies on some deep results of Elliott and Lin. We have

$$
\mathcal{A}_{\Theta} \simeq \mathcal{A}_{(-\Theta)} .
$$

Sketch of the proof. When $d(\Theta) \in\{1,2\}$, it follws from simple considerations that $\Theta \sim(-\Theta)$, hence $\mathcal{A}_{\Theta} \simeq \mathcal{A}_{(-\Theta)}$ by (a). Assume now $d(\Theta) \in\{3,4\}$, i. e $\mathcal{A}_{\Theta}$ is simple with a unique trace by Slawny's theorem. Then, as shown in [Lin], $\mathcal{A}_{\Theta}$ is a unital inductive limit of of direct sums of four circle algebras. Consequently, using the classification theorem of Elliott in [Ell3] (see also [NT]), the ordered $K_{0}$-group (cf. [Bla]) with distinguished order unit is a complete isomorphism invariant for simple noncommutative 3-tori. Now let $\mathcal{A}_{\Theta}^{o p}$ denote the $\mathrm{C}^{*}$-algebra opposite to $\mathcal{A}_{\Theta}$, i. e. equipped with reverse multiplication. By construction, it is clear that $\mathcal{A}_{\Theta}$ and $\mathcal{A}_{\Theta}^{o p}$ have the same invariant. On the other hand, it is quite obvious that $\mathcal{A}_{\Theta}^{o p}$ is isomorphic to $\mathcal{A}_{(-\Theta)}$. Hence we deduce that $\mathcal{A}_{\Theta} \simeq \mathcal{A}_{(-\Theta)}$ as desired.

By using (b), (c) and (d) we obtain the following classification result.

Theorem. We have $\mathcal{A}_{\Theta} \simeq \mathcal{A}_{\tilde{\Theta}} \Longleftrightarrow \Theta \approx \tilde{\Theta}$.

Concerning the classification of noncommutative smooth 3-tori, regarded as locally convex topological algebras in a natural way, a deep result from [CEGJ, BCEN, Bre], relying on Connes' cyclic cohomology, says that

$$
\mathcal{A}_{\Theta}^{\infty} \simeq \mathcal{A}_{\tilde{\Theta}}^{\infty} \Longleftrightarrow \Theta \sim \tilde{\Theta} .
$$

As alluded to in [Bre], this classification result, combined with (d), has the following consequence. Assume that $d(\Theta)=4$, i. e. $\Theta$ is totally irrational. Then we have $\mathcal{A}_{\Theta} \simeq \mathcal{A}_{(-\Theta)}$, while $\mathcal{A}_{\Theta}^{\infty}$ is not isomorphic to $\mathcal{A}_{(-\Theta)}^{\infty}$. This means that $\mathcal{A}_{\Theta}$ is then an example of a simple $\mathrm{C}^{*}$-algebra with two different differential stuctures.

4.9 We shall briefly explain how rational noncommutative 3-tori are easily classified and how any such algebra is isomorphic to a $\mathrm{C}^{*}$-subalgebra of some matrix algebra over $C\left(\mathbb{T}^{3}\right)$ (and thereby subhomogeneous). 
We assume that $\Theta$ is rational, $\theta_{j}=\frac{p_{j}}{q_{j}} \in \mathbb{Q}, j=1,2,3$, and without loss of generality (using 4.1) we also assume that $1 \leq\left|p_{j}\right| \leq q_{j}$ and $\left(p_{j}, q_{j}\right)=1$, $j=1,2,3$.

We set $q=q(\Theta)=1$. c. m. $\left(q_{1}, q_{2}, q_{3}\right)$ and $\tilde{\Theta}=\left(0,0, \frac{1}{q}\right)$. A simple numbertheoretical argument gives that $\Theta \sim \tilde{\Theta}$. Hence we have

$$
\mathcal{A}_{\Theta} \simeq \mathcal{A}_{\tilde{\Theta}} \simeq C(\mathbb{T}) \otimes \mathcal{A}_{\frac{1}{q}}
$$

the first isomorphism being given by 4.8 (a) and the second being the natural one determined by sending the canonical generators $\tilde{U}_{1}, \tilde{U}_{2}$ and $\tilde{U}_{3}$ of $\mathcal{A}_{\tilde{\Theta}}$ onto $1 \otimes U, 1 \otimes V$ and $W \otimes 1$, respectively, where $U$ and $V$ denote the canonical generators of $\mathcal{A}_{\frac{1}{q}}$ and $\mathrm{W}$ the canonical generator of $C(\mathbb{T})$. We refer to [Bre] for an analogous result for rational noncommutative tori of arbitrary dimension. It follows easily from this result that rational noncommutative 3 -tori are classified by $q(\Theta)$.

If $q=1$, i. e. $\Theta=(1,1,1) \sim \mathbf{0}$, then $\mathcal{A}_{\Theta} \simeq C\left(\mathbb{T}^{3}\right)$. Assume now that $q \geq 2$. Then it is known ([Bel2, CEY]) that $\mathcal{A}_{\frac{1}{q}}$ is isomorphic to the $\mathrm{C}^{*}$-subalgebra of $C\left(\mathbb{T}^{2}\right) \otimes M_{q}(\mathbb{C}) \simeq C\left(\mathbb{T}^{2}, M_{q}(\mathbb{C})\right)$ generated by the two $M_{q}(\mathbb{C})$-valued functions $\tilde{U}$ and $\tilde{V}$ on $\mathbb{T}^{2}$ defined by

$$
\begin{aligned}
& \tilde{U}\left(z_{1}, z_{2}\right)=z_{1}\left[\begin{array}{ccccc}
0 & 1 & 0 & \ldots & 0 \\
\ldots & \ldots & \ldots & \ldots & \ldots \\
0 & 0 & 0 & \ldots & 1 \\
1 & 0 & 0 & \ldots & 0
\end{array}\right], \\
& \tilde{V}\left(z_{1}, z_{2}\right)=z_{2}\left[\begin{array}{ccccc}
1 & 0 & 0 & \ldots & 0 \\
0 & e^{i 2 \pi \frac{1}{q}} & 0 & \ldots & 0 \\
\ldots & \ldots & \ldots & \ldots & \ldots \\
0 & 0 & 0 & \ldots & e^{i 2 \pi \frac{q-1}{q}}
\end{array}\right] .
\end{aligned}
$$

It follows therefore that $\mathcal{A}_{\tilde{\Theta}}$ is isomorphic to the $\mathrm{C}^{*}$-subalgebra of $C(\mathbb{T}) \otimes C\left(\mathbb{T}^{2}, M_{q}(\mathbb{C})\right) \simeq C\left(\mathbb{T}^{3}, M_{q}(\mathbb{C})\right)$ generated by the three $M_{q}(\mathbb{C})$-valued functions $\hat{U}_{1}, \hat{U}_{2}$ and $\hat{U}_{3}$ on $\mathbb{T}^{3}$ defined by

$$
\begin{aligned}
& \hat{U}_{1}\left(z_{1}, z_{2}, z_{3}\right)=\tilde{U}\left(z_{1}, z_{2}\right), \\
& \hat{U}_{2}\left(z_{1}, z_{2}, z_{3}\right)=\tilde{V}\left(z_{1}, z_{2}\right), \\
& \hat{U}_{3}\left(z_{1}, z_{2}, z_{3}\right)=z_{3} I_{q} \quad\left(I_{q} \text { denoting the } q \times q \text { identity matrix }\right),
\end{aligned}
$$

via an isomorphism sending $\tilde{U}_{j}$ onto $\hat{U}_{j}, j=1,2,3$.

Combining this with the result above, we obtain that $\mathcal{A}_{\Theta}$ is isomorphic to the $\mathrm{C}^{*}$-subalgebra of $C\left(\mathbb{T}^{3}, M_{q}(\mathbb{C})\right) \simeq M_{q}\left(C\left(\mathbb{T}^{3}\right)\right)$ generated by $\hat{U}_{1}, \hat{U}_{2}$ and $\hat{U}_{3}$, as desired. 
4.10 Let I denote a closed box in $\mathbb{R}^{3}$ equipped with its usual topology. It follows from a more general result on continuous fields of $\mathrm{C}^{*}$-algebras arising from varying cocycles on amenable groups due to Rieffel ([Rie3], see also [ENN]) that the family $\left\{\mathcal{A}_{\Theta}\right\}_{\Theta \in \mathbf{I}}=\left\{C_{r}^{*}\left(\mathbb{Z}^{3}, \sigma_{\Theta}\right)\right\}_{\Theta \in \mathbf{I}}$ is a continuous field of $\mathrm{C}^{*}$-algebras. Its associated $\mathrm{C}^{*}$-algebra $\mathcal{A}_{\mathbf{I}, \sigma}$ of continuous sections may be described as the twisted $\mathrm{C}^{*}$-crossed product $C^{*}\left(C(\mathbf{I}), \mathbb{Z}^{3}\right.$, id,$\left.\sigma_{\mathrm{I}}\right)$, where id denotes the trivial action of $\mathbb{Z}^{3}$ on $C(\mathbf{I})$ and $\sigma_{\mathbf{I}}$ denotes the 2-cocycle on $\mathbb{Z}^{3}$ with values in the unitaries of $C(\mathbf{I})$ defined by

$$
\left[\sigma_{\mathbf{I}}(\mathbf{x}, \mathbf{y})\right](\Theta)=\sigma_{\Theta}(\mathbf{x}, \mathbf{y}) .
$$

Indeed, for each $\Theta \in \mathbf{I}$, there exists a canonical homomorphism $\pi_{\Theta}$ from $C^{*}\left(C(\mathbf{I}), \mathbb{Z}^{3}\right.$, id,$\left.\sigma_{\mathbf{I}}\right)$ onto $C^{*}\left(\mathbb{Z}^{3}, \sigma_{\Theta}\right) \simeq C_{r}^{*}\left(\mathbb{Z}^{3}, \sigma_{\Theta}\right)$ determined by

$$
\pi_{\Theta}\left(a \lambda_{\mathbf{I}}(\mathbf{m})\right)=a(\Theta) \lambda_{\Theta}(\mathbf{m}), \quad a \in C(\mathbf{I}), \mathbf{m} \in \mathbb{Z}^{3},
$$

(where $\lambda_{\mathbf{I}}(\mathbf{m})$ denotes the canonical unitary in $C^{*}\left(C(\mathbf{I}), \mathbb{Z}^{3}\right.$,id, $\sigma_{\mathbf{I}}$ ) associated to each $\left.\mathbf{m} \in \mathbb{Z}^{3}\right)$.

The map $\pi$ from $C^{*}\left(C(\mathbf{I}), \mathbb{Z}^{3}\right.$, id, $\left.\sigma_{\mathbf{I}}\right)$ onto $\mathcal{A}_{\mathbf{I}, \sigma}$ defined by

$$
\pi(A)=\left\{\pi_{\Theta}(A)\right\}_{\Theta \in \mathbf{I}}
$$

gives then the desired isomorphism.

We remark that one may avoid introducing twisted $\mathrm{C}^{*}$-crossed products explicitely by adapating the approach described by Bellissard ([Bel6]) in the $2 \mathrm{D}$ case. There is also another approach due to Anderson and Paschke ([AP]) in the $2 \mathrm{D}$ case which is related to the group $\mathrm{C}^{*}$-algebra of the classical 3-dimensional discrete Heisenberg group. This approach may be carried over to the 3D case and is then related to the group $\mathrm{C}^{*}$-algebra of some 6-dimensional discrete Heisenberg group. In fact, there is a subtlety here in that choosing different, but cohomologous cocycles does not necessarily lead to isomorphic Heisenberg groups. As it would take us too far afield from our main theme to explain this more precisely, we will deal with these matters in a separate work ([Bed3]).

\section{Some spectral properties of 3D discrete mag- netic Laplacians}

We continue to use the notation introduced in the previous sections. Let $\Theta, \mathbf{c} \in \mathbb{R}^{3}$. We set

$$
S(\Theta, \mathbf{c})=S p\left(\Delta_{\Theta, \mathbf{c}}\right) \text { and } S(\Theta)=S p\left(\Delta_{\Theta}\right)=S(\Theta,(1,1,1)) .
$$


5.1 Proposition. Let $\tilde{\Theta}=\Theta\left(\bmod \mathbb{Z}^{3}\right)$ and $\tilde{\mathbf{c}}=\left( \pm c_{1}, \pm c_{2}, \pm c_{3}\right)$. Then we have

$$
S(\tilde{\Theta}, \mathbf{c})=S(\Theta, \mathbf{c})=S(\Theta, \tilde{\mathbf{c}})
$$

Proof. The first equality follows easily from 4.1. Let $\gamma=( \pm 1, \pm 1, \pm 1) \in \mathbb{T}^{3}$. From 4.4 we get $\alpha_{\gamma}\left(\Delta_{\Theta, \mathbf{c}}\right)=\Delta_{\Theta, \tilde{\mathbf{c}}}$ and the second equality follows readily.

5.2 Proposition. Let $a=\left\|\Delta_{\Theta, \mathbf{c}}\right\|$. Then $S(\Theta, \mathbf{c})$ is a closed subset of $[-a, a]$ which is symmetric around 0 and contains $\pm a$.

Proof. Using 5.1 with $\tilde{\mathbf{c}}=-\mathbf{c}$ we have

$$
S(\Theta, \mathbf{c})=S p\left(\Delta_{\Theta,(-\mathbf{c})}\right)=S p\left(-\Delta_{\Theta, \mathbf{c}}\right)=-S(\Theta, \mathbf{c}) .
$$

As $\Delta_{\Theta, c}$ is bounded and self-adjoint, the result follows from elementary operator theory.

An obvious upper bound for $\left\|\Delta_{\Theta, \mathbf{c}}\right\|$ is $2\left(\left|c_{1}\right|+\left|c_{2}\right|+\left|c_{3}\right|\right)$. Determining better estimates for $\left\|\Delta_{\Theta, \mathbf{c}}\right\|$, or just for $\left\|\Delta_{\Theta}\right\|$, should be a challenging problem, as it is in the $2 \mathrm{D}$ case (see [BVZ]).

5.3 Let $\theta, c \in \mathbb{R}, c \neq 0$. In the $2 \mathrm{D}$ case, we have

$$
S p\left(\Delta_{(-\theta), c}\right)=S p\left(\Delta_{\theta, c}\right)=c S p\left(\Delta_{\theta, \frac{1}{c}}\right),
$$

the second equality being usually called Aubry (or André-Aubry) duality, cf. [AS, Bel2, Bel4]. A somewhat analagous result in 3D may be formulated as follows.

Proposition. Let $\check{\Theta}=\left( \pm \theta_{p(1)}, \pm \theta_{p(2)}, \pm \theta_{p(3)}\right)$ and $\breve{\mathbf{c}}=\left(c_{p(1)}, c_{p(2)}, c_{p(3)}\right)$, where $p$ is a permutation of $\{1,2,3\}$. Then we have

$$
S(\check{\Theta}, \breve{\mathbf{c}})=S(\Theta, \mathbf{c}) .
$$

Especially, we have

$$
S(\check{\Theta})=S(\Theta) \text {. }
$$

Proof. The second assertion follows from the first by setting $\mathbf{c}=(1,1,1)$. The the first assertion is proved by the following three steps. We denote by $A$ the standard matrix of the linear map $\Theta \longrightarrow \check{\Theta}$ from $\mathbb{R}^{3}$ into $\mathbb{R}^{3}$, so $A \in G L(3, \mathbb{Z})$. 
i) Assume first that $\operatorname{det}(A)=1$. Then one checks easily that the isomorphism $\iota_{A}$ from $\mathcal{A}_{\Theta}$ onto $\mathcal{A}_{A \Theta}=\mathcal{A}_{\check{\Theta}}$ introduced in 4.8 (a) sends $\Delta_{\Theta, \mathbf{c}}$ to $\Delta_{\check{\Theta}, \breve{c}}$. Hence we get $S(\check{\Theta}, \breve{\mathbf{c}})=S(\Theta, \mathbf{c})$ in this case.

ii) Secondly, we observe that there exists an obvious natural anti-isomorphism from $\mathcal{A}_{\Theta}$ onto $\mathcal{A}_{(-\Theta)}$ (by identifying $\mathcal{A}_{(-\Theta)}$ with the opposite algebra of $\mathcal{A}_{\Theta}$ ) which sends $\Delta_{\Theta, \mathbf{c}}$ to $\Delta_{(-\Theta), \mathbf{c}}$. As anti-isomorphisms also preserve spectra, we get $S(-\Theta, \mathbf{c})=S(\Theta, \mathbf{c})$.

iii) Assume at last that $\operatorname{det}(A)=-1$, so $\operatorname{det}(-A)=1$. Then, writing $(-\check{\Theta})=(-A) \Theta$ and using i $)$, we get $S(-\check{\Theta}, \breve{\mathbf{c}})=S(\Theta, \mathbf{c})$. On the other hand, using ii), we have $S(-\check{\Theta}, \breve{\mathbf{c}})=S(\check{\Theta}, \breve{\mathbf{c}})$. Hence we get $S(\check{\Theta}, \breve{\mathbf{c}})=S(\Theta, \mathbf{c})$ also in this case.

The connection with Aubry duality is more apparent when (at least) two of the components of $\Theta$ are equal $\left(\bmod \mathbb{Z}^{3}\right)$. Assume for example that $\theta_{1}=\theta_{2}$. By choosing $p$ to be the permutation interchanging 1 and 2, we get then

$$
\begin{gathered}
S(\Theta, \mathbf{c})=S\left(\Theta,\left(c_{2}, c_{1}, c_{3}\right)\right), \text { and therefore } \\
S(\Theta,(1, c, d))=c S(\Theta,(1,1 / c, d / c)), c \neq 0 .
\end{gathered}
$$

5.4 By using Propositions 5.1, 5.2 and 5.3, we see that we may restrict our attention to the case when $0 \leq \theta_{1} \leq \theta_{2} \leq \theta_{3} \leq 1 / 2$ and $c_{j} \geq 0, j=1,2,3$.

5.5 Proposition. Let $\mathbf{c} \neq \mathbf{0}$. Then $\Delta_{\Theta, \mathbf{c}}$ has no point spectrum. Especially $S(\Theta$, c) has no isolated points, $i$. e. is a perfect set.

Proof. We adapt an argument due to Delyon and Souillard ([DS]) given in the context of 1D random discrete Schrödinger operators (see also [CFKS]).

Let $J$ be a finite, nonempty subset of $\mathbb{Z}^{3}$ and denote by $P_{J}$ the orthogonal projection from $\ell^{2}\left(\mathbb{Z}^{3}\right)$ onto $\ell^{2}(J)$. Let $W \in \mathcal{W}_{\Theta}$.

Since $\tau(W)=\tau\left(\lambda_{\Theta}(\mathbf{x})^{*} W \lambda_{\Theta}(\mathbf{x})\right)=<W \delta_{\mathbf{x}}, \delta_{\mathbf{x}}>$ for all $\mathbf{x} \in \mathbb{Z}^{3}$, we get easily

$$
\tau(W)=\frac{1}{\#(J)} \operatorname{Tr}\left(P_{J} W\right) .
$$

Set now $J(n)=\{-n, \ldots, 0, \ldots, n\}$ and $P_{n}=P_{J(n)}$ for each $n \in \mathbb{N}$.

Assume (for contradiction) that $\lambda \in \mathbb{R}$ is an eigenvalue for $\Delta_{\Theta, \mathbf{c}}$. Denote by $Q \in \mathcal{W}_{\Theta}$ the spectral projection of $\Delta_{\Theta, \mathrm{c}}$ associated to $\{\lambda\}$, so $Q \neq 0$.

Let $n \in \mathbb{N}, n \geq 3$. Using the definition of $\Delta_{\Theta, \mathbf{c}}$ and the assumption that $\mathbf{c}$ has (at least) one nonzero component, it is not difficult to see that

$$
\operatorname{dim}\left(P_{n} Q\left(\ell^{2}\left(\mathbb{Z}^{3}\right)\right) \leq \#(J(n) \backslash J(n-2)) .\right.
$$


(One can achieve a better upper bound here, but this one suffices for our purposes.) Hence we get

$$
\begin{aligned}
0 \leq \tau(Q) & =\frac{1}{\#(J(n)}\left|\operatorname{Tr}\left(P_{n} Q\right)\right| \leq \frac{1}{\#(J(n)} \operatorname{dim}\left(P_{n} Q\left(\ell^{2}\left(\mathbb{Z}^{3}\right)\right)\right. \\
& \leq \frac{\#(J(n) \backslash J(n-2))}{\#\left(J_{n}\right)} .
\end{aligned}
$$

But, as

$$
\frac{\#(J(n) \backslash J(n-2))}{\#\left(J_{n}\right)}=\frac{(2 n+1)^{3}-(2 n-3)^{3}}{(2 n+1)^{3}} \rightarrow 0 \text { as } n \rightarrow \infty,
$$

this implies that $\tau(Q)=0$, thus $Q=0$ as $\tau$ is faithful. We have obtained a contradiction, and the result follows.

With some more work, the same idea can be pursued to show that this proposition also holds for any nonzero selfadjoint operator lying in the *-algebra generated by $\left\{\lambda_{\Theta}(\mathbf{x}) \mid \mathbf{x} \in \mathbb{Z}^{3}\right\}$.

5.6 Let $\left\{\Gamma_{n}\right\}_{n \geq 1}$ be any Fölner sequence for $\mathbb{Z}^{3}$ (cf. e. g. [Bed2]). For example, we may take $\Gamma_{n}=\{-n, \ldots, 0, \ldots, n\}^{3}$ or $\Gamma_{n}=\{0,1, \ldots, n\}^{3}$. Set $\mathcal{H}_{n}=\ell^{2}\left(\Gamma_{n}\right)$ and let $P_{n}$ be the orthogonal projection from $\ell^{2}\left(\mathbb{Z}^{3}\right)$ onto $\ell^{2}\left(\mathcal{H}_{n}\right)$. Let $T \in \mathcal{W}_{\Theta}$.

We denote by $\mu_{T}^{n}$ the spectral measure of the compression $T_{n}=P_{n} T_{\mid \mathcal{H}_{n}}$ with respect to the normalized trace on $\mathcal{B}\left(\mathcal{H}_{n}\right)$, i. e.

$\mu_{T}^{n}(S)=\frac{1}{\#\left(\Gamma_{n}\right)} \cdot\left(\right.$ number of eigenvalues of $T_{n}$ in $\left.S\right), \quad$ (multiplicities counted).

The IDS (integrated density of states) of $T$ at $\lambda \in \mathbb{R}$ (with respect to $\left\{P_{n}\right\}$ ), see [AS, CFKS] for an explanation of this terminology, is defined by

$$
N_{T}(\lambda)=\lim _{n \rightarrow \infty} \mu_{T}^{n}((-\infty, \lambda])
$$

whenever this limit exists.

On the other hand, let $\mu_{T}^{\tau}$ denote the spectral measure of $W$ with respect to $\tau$, so

$$
\mu_{T}^{\tau}(S)=\tau\left(E_{T}(S)\right), S \text { Borel subset of } \mathbb{R},
$$

where $E_{T}$ denotes the projection-valued measure associated to $T$ in $\mathcal{W}_{\Theta}$.

The spectrum distribution function of $T$ ( in the terminology of [Shu]) is defined by

$$
D_{T}(\lambda)=\mu_{T}^{\tau}((-\infty, \lambda]), \lambda \in \mathbb{R} .
$$


It follows from [Bed2] that the Borel probability measure $\mu_{T}^{n}$ converges weakly to $\mu_{T}^{\tau}$ as $n \rightarrow \infty$. From a classical result in probability theory, this implies that $N_{T}(\lambda)$ exists and is equal to $D_{T}(\lambda)$ whenever $\mu_{T}^{\tau}(\{\lambda\})=0$, i. e. whenever $\lambda$ is not an eigenvalue of $T$, or, equivalently, whenever $D_{T}$ is continuous at $\lambda$. Taking into account Proposition 5.5, the next proposition clearly follows.

Proposition. The IDS of $\Delta_{\Theta, \mathbf{c}}$ exists for all $\lambda \in \mathbb{R}$. It is continuous on $\mathbb{R}$ and equal to the spectrum distribution function of $\Delta_{\Theta, \mathbf{c}}$.

It is elementary to see that $S(\Theta, \mathbf{c})$ may described as the support of the spectral measure of $\Delta_{\Theta, c}$ with respect to $\tau$ (cf. [Bed2]), i. e. as the set of nonconstancy points of the spectrum distribution function of $\Delta_{\Theta, c}$. Hence, the above result means that $S(\Theta$, c $)$ may be approximated numerically by computing the spectrum of $P_{n} \Delta_{\Theta, \mathbf{c} \mid \mathcal{H}_{n}}$ for large $n$ (cf. [Arv, Bed2]). However, it seems quite hard to do this in an effective way, the size of the resulting matrices being of order $n^{3} \times n^{3}$. We shall describe in 5.7 and 5.11 some other numerical methods which seem potentially more efficient.

5.7 Proposition. Let $\Theta$ be rational. Then every selfadjoint operator in $\mathcal{A}_{\Theta}$ (so especially $\left.\Delta_{\Theta, \mathrm{c}}\right)$ has a band-spectrum.

Proof. This follows from 4.9. If we use the notation introduced there and let $q=q(\Theta)$, then every element of $\mathcal{A}_{\Theta}$ may be represented as a $q \times q$ matrix over $C\left(\mathbb{T}^{3}\right)$. Hence the spectrum of every selfadjoint operator in $\mathcal{A}_{\Theta}$ must consist of at most $q$ bands. (This can also be deduced using the main result of [Ell2] mentionned in 4.6).

For concretely given $\Theta \in \mathbb{Q}^{3}$ and $\mathbf{c} \in \mathbb{R}^{3}$, it is possible to trace the isomorphism from $\mathcal{A}_{\Theta}$ onto $M_{q}\left(C\left(\mathbb{T}^{3}\right)\right)$ described in 4.9, and thereby obtain a (somewhat complicated) formula for the representant of $\Delta_{\Theta, \mathrm{c}}$ in $M_{q}\left(C\left(\mathbb{T}^{3}\right)\right)$, from which $S(\Theta$, c $)$ can then be approximated numerically. However, we have not so far found an effective procedure which implements this process in general, and each case must be computed separately. The simple case when $q(\Theta)=2$ can be done by hand, and one obtains e. g.

$$
\begin{aligned}
& S\left(\left(0,0, \frac{1}{2}\right)\right)=[-2(1+\sqrt{2}), 2(1+\sqrt{2})], \\
& S\left(0, \frac{1}{2}, \frac{1}{2}\right)=[-2 \sqrt{5}, 2 \sqrt{5}] \\
& S\left(\frac{1}{2}, \frac{1}{2}, \frac{1}{2}\right)=[-2 \sqrt{3}, 2 \sqrt{3}] .
\end{aligned}
$$

In view of the above remark, the next result means that it should then be possible to compute better and better approximations of $S(\Theta, \mathbf{c})$ for a given nonrational $\Theta$, but so far only in an quite ineffective way. We hope to come back to these numerical considerations in a later work. 
5.8 Proposition. Let $\mathbf{c} \in \mathbb{R}^{3}$ and let $\mathbf{I}$ denote a closed box in $\mathbb{R}^{3}$ containing $\Theta$, equipped with its usual topology. Then the family $\{S(\Theta, \mathbf{c})\}_{\Theta \in \mathbf{I}}$ is continuous in the following sense : For each open set $U \subseteq \mathbb{R}$, the sets $\{\Theta \in \mathbf{I} \mid S(\Theta, \mathbf{c}) \subseteq U\}$ and $\{\Theta \in \mathbf{I} \mid S(\Theta, \mathbf{c}) \cap U \neq \emptyset\}$ are both open subsets of $\mathbf{I}$.

Proof. Since the family $\left\{\mathcal{A}_{\Theta}\right\}_{\Theta \in \mathbf{I}}$ is a continuous field of $\mathrm{C}^{*}$-algebras, cf. 4.10, and $\left\{\Delta_{\Theta, \mathbf{c}}\right\}_{\mathrm{I}}$ is a normal continuous section of it, the result follows from [Ell1]. Alternatively, one can proceed as in [Bel6].

It is easy to see that the map c $\longrightarrow S(\Theta, \mathbf{c})$ is Hausdorff-continuous. It is quite possible that the map $\Theta \longrightarrow S(\Theta, \mathbf{c})$ is also Hausdorff-continuous (as its analogue is in the 2D case, cf. [CEY], see also [HR]). We shall give an indication of this in 5.11. The continuity property expressed in the proposition above is weaker than Hausdorff continuity. It may be rephrased as follows: whenever $\Theta_{k}$ is a sequence in $\mathbb{R}^{3}$ converging to $\Theta$, we have

$$
S(\Theta, \mathbf{c})=\left\{\lambda \in \mathbb{R} \mid \exists \lambda_{k} \in S\left(\Theta_{k}, \mathbf{c}\right) \text { for each k, s. t. } \lambda_{k} \rightarrow \lambda\right\} .
$$

5.9 In the simple case when $\Theta$ is parallel to one of the axes, we can say more about $S(\Theta, \mathbf{c})$. Without loss of generality (using 5.3), we only consider the third axe.

Proposition. Let $\theta \in \mathbb{R}$ and set $\Theta=(0,0, \theta)$. Assume that $c_{3} \neq 0$. Then $S(\Theta, \mathbf{c})$ is not a Cantor set and we have

$$
S(\Theta)=\left[-\left(2+\left\|\Delta_{\theta}\right\|\right),\left(2+\left\|\Delta_{\theta}\right\|\right)\right] .
$$

Proof. Let $U$ and $V$ denote the canonical generators of $\mathcal{A}_{\theta}$ and $W$ denotes the canonical generator of $C(\mathbb{T})$. Then there exists an obvious isomorphism $\pi$ from $\mathcal{A}_{\Theta}$ onto $C(\mathbb{T}) \otimes \mathcal{A}_{\theta}$ satisfying

$$
\pi\left(U_{1}\right)=1 \otimes U, \pi\left(U_{2}\right)=1 \otimes V, \pi\left(U_{3}\right)=W \otimes 1 .
$$

Hence we get

$$
\pi\left(\Delta_{\Theta, \mathbf{c}}\right)=1 \otimes\left[c_{1}\left(U^{*}+U\right)+c_{2}\left(V^{*}+V\right)\right]+c_{3}\left(W^{*}+W\right) \otimes 1,
$$

which implies that

$$
\begin{aligned}
S(\Theta, \mathbf{c}) & =S p\left(c_{1}\left(U^{*}+U\right)+c_{2}\left(V^{*}+V\right)\right)+c_{3} S p\left(W^{*}+W\right) \\
& =S p\left(c_{1}\left(U^{*}+U\right)+c_{2}\left(V^{*}+V\right)\right)+\left[-2\left|c_{3}\right|, 2\left|c_{3}\right|\right]
\end{aligned}
$$

and the first assertion clearly follows. Further, this gives

$$
S(\Theta)=S p\left(\Delta_{\theta}\right)+[-2,2] .
$$

Now we have $\left\|\Delta_{\theta}\right\| \leq 4$ and $S p\left(\Delta_{\theta}\right) \subseteq\left[-\left\|\Delta_{\theta}\right\|,\left\|\Delta_{\theta}\right\|\right]$ with $\pm\left\|\Delta_{\theta}\right\| \in S p\left(\Delta_{\theta}\right)$ (from the 2D analogue of Proposition 5.2). Further, we know from [CEY],p.232, that $0 \in S p\left(\Delta_{\theta}\right)$. The last assertion is then easily deduced. 
The case when $\Theta=(0,0, \theta)$ and $c_{3}=0$ is essentially the $2 \mathrm{D}$ problem. For example, we have

$$
S\left((0,0, \theta),\left(c_{1}, c_{2}, 0\right)\right)=c_{1} S p\left(\Delta_{\theta, c_{2} / c_{1}}\right) \text { whenever } c_{1} \neq 0,
$$

and this set is then "generically" a Cantor set, cf. [BS].

The above proposition illustrates that the gap labelling theory (see [Bel1, Bel5]) is not always useful. This theory predicts that when $\Theta=(0,0, \theta)$, the possible gap levels for $\Delta_{\Theta}$ are given by numbers in $(\mathbb{Z}+\theta \mathbb{Z}) \cap[0,1]$ (using 4.6). On the other hand, the only gap levels of $\Delta_{\Theta}$ in this case are 0 and 1 , since $S(\Theta)$ is then connected, as shown above. Note that we are within the degenerate case for this choice of $\Theta$. It is conceivable that the gap labelling theory may turn to be useful only when $\Theta$ is nondegenerate.

5.10 We shall now etablish a connection between 3D discrete magnetic Laplacians and some 2D discrete almost periodic magnetic Schrödinger operators, which we call 2D almost Mathieu operators in view of the obvious analogy with such 1D operators. We first define these operators.

Let $\Theta, \mathbf{c} \in \mathbb{R}^{3}$ be given. We denote by $U$ and $V$ the canonical generators of $\mathcal{A}_{\theta_{3}}$ acting on $\ell^{2}\left(\mathbb{Z}^{2}\right)$. For each $\beta \in \mathbb{R}$ we define $F_{\beta}$ to be the multiplication operator on $\ell^{2}\left(\mathbb{Z}^{2}\right)$ associated to the real function on $\mathbb{Z}^{2}$ given by

$$
\left(m_{1}, m_{2}\right) \longrightarrow 2 \cos \left(2 \pi\left(m_{1} \theta_{2}-m_{2} \theta_{1}+\beta\right)\right) .
$$

We define then the $2 D$ almost Mathieu operator on $\ell^{2}\left(\mathbb{Z}^{2}\right)$ associated to $\Theta, \mathbf{c}, \beta$ as the operator given by

$$
H_{\Theta, \mathbf{c}, \beta}=c_{1}\left(U^{*}+U\right)+c_{2}\left(V^{*}+V\right)+c_{3} F_{\beta} .
$$

To see more clearly the analogy with the 1D almost Mathieu operators defined in Section 2, we observe that if $\mathbf{c}=(1, c, d)$, we have

$$
H_{\Theta, \mathbf{c}, \beta}=\Delta_{\theta_{3}, c}+d F_{\beta} .
$$

To etablish the desired connection, let us now introduce the unitary operator $W_{\beta}$ on $\ell^{2}\left(\mathbb{Z}^{2}\right)$ defined as the multiplication operator associated to the complex function on $\mathbb{Z}^{2}$ given by

$$
\left(m_{1}, m_{2}\right) \longrightarrow e^{i 2 \pi\left(m_{1} \theta_{2}-m_{2} \theta_{1}+\beta\right)} .
$$

Then we clearly have $F_{\beta}=W_{\beta}^{*}+W_{\beta}$. Further, one checks that $U, V$ and $W_{\beta}$ satisfy the relations

$$
\left\{\begin{array}{l}
U V=e^{i 2 \pi \theta_{3}} V U, \\
V W_{\beta}=e^{i 2 \pi \theta_{1}} W_{\beta} V, \\
W_{\beta} U=e^{i 2 \pi \theta_{2}} U W_{\beta} .
\end{array}\right.
$$


Using the universal property of $\mathcal{A}_{\Theta}$, there exists a homomorphism $\pi_{\beta}$ from $\mathcal{A}_{\Theta}$ onto $C^{*}\left(U, V, W_{\beta}\right)$ such that

$$
\pi_{\beta}\left(U_{1}\right)=U, \pi_{\beta}\left(U_{2}\right)=V, \pi_{\beta}\left(U_{3}\right)=W_{\beta} .
$$

Then we get

$$
\pi_{\beta}\left(\Delta_{\Theta, \mathbf{c}}\right)=H_{\Theta, \mathbf{c}, \beta}
$$

As briefly described in $4.7, \mathcal{A}_{\Theta}$ may be written as a twisted crossed product of $C(\mathbb{T})$ by a twisted action of $\mathbb{Z}^{2}$, and $\pi_{\beta}$ is then a representation of this crossed product obtained by a well-known procedure.

Our interest in 2D almost Mathieu operators stems from the following result, which is clearly analogous to one of the results recalled in Section 2.

Proposition. We have

$$
S(\Theta, \mathbf{c})= \begin{cases}S p\left(H_{\Theta, \mathbf{c}, \beta}\right) & \text { if } \Theta \text { is nondegenerate } \\ \bigcup_{\beta \in[0,1]} S p\left(H_{\Theta, \mathbf{c}, \beta}\right) & \text { if } \Theta \text { is degenerate. }\end{cases}
$$

Proof. The proof goes along the same lines as in the 2D case (see [CEY],

p. 235). If $\Theta$ is nondegenerate, then $\mathcal{A}_{\Theta}$ is simple by Slawny's theorem and $\pi_{\beta}$ is therefore an isomorphism, so $S(\Theta, \mathbf{c})=S p\left(H_{\Theta, \mathbf{c}, \beta}\right)$ as desired.

Assume now that $\Theta$ is degenerate. It is then enough to show that the family $\left\{\pi_{\beta}\right\}_{\beta \in[0,1]}$ of representations of $\mathcal{A}_{\Theta}$ is separating, i. e. that

$$
\mathcal{J}:=\bigcap_{\beta \in[0,1]} \operatorname{ker} \pi_{\beta}=\{0\},
$$

since this implies that

$$
S(\Theta, \mathbf{c})=\overline{\bigcup_{\beta \in[0,1]} S p\left(H_{\Theta, \mathbf{c}, \beta}\right)}=\bigcup_{\beta \in[0,1]} S p\left(H_{\Theta, \mathbf{c}, \beta}\right),
$$

the last equality being not hard to see.

To show that $\mathcal{J}=\{0\}$, we appeal to the action $\alpha$ of $\mathbb{T}^{3}$ on $\mathcal{A}_{\Theta}$ defined in 4.4: as $\alpha$ is ergodic, it is then enough to show that $\mathcal{J}$ is invariant under $\alpha$.

For $z_{1}, z_{2} \in \mathbb{T}$, let $V_{z_{1}, z_{2}}$ denote the unitary operator on $\ell^{2}\left(\mathbb{Z}^{2}\right)$ defined as the multiplication operator associated to the complex function on $\mathbb{Z}^{2}$ given by

$$
\left(m_{1}, m_{2}\right) \longrightarrow z_{1}^{m_{1}} z_{2}^{m_{2}} .
$$

For $\beta \in \mathbb{R}$, we may then define a representation $\pi_{z_{1}, z_{2}, \beta}$ of $\mathcal{A}_{\Theta}$ on $\ell^{2}\left(\mathbb{Z}^{2}\right)$ by

$$
\pi_{z_{1}, z_{2}, \beta}=\left(\operatorname{Ad} V_{z_{1}, z_{2}}\right) \circ \pi_{\beta} .
$$


Now, if $\gamma \in \mathbb{T}^{3}$ and we write $\gamma_{3}=e^{i 2 \pi \omega}$ for some $\omega \in \mathbb{R}$, then one checks that

$$
\pi_{z_{1}, z_{2}, \beta} \circ \alpha_{\gamma}=\pi_{z_{1} \gamma_{1}, z_{2} \gamma_{2}, \beta+\omega}
$$

So if $A \in \mathcal{J}$, we get

$$
\begin{aligned}
\pi_{\beta}\left(\alpha_{\gamma}(A)\right) & =\pi_{1,1, \beta}\left(\alpha_{\gamma}(A)\right) \\
& =\pi_{\gamma_{1}, \gamma_{2}, \beta+\omega}(A) \\
& =\left(A d V_{\gamma_{1}, \gamma_{2}}\right)\left(\pi_{\beta+\omega}(A)\right) \\
& =0
\end{aligned}
$$

for all $\beta \in \mathbb{R}$, which implies that

$$
\alpha_{\gamma}(A) \in \bigcap_{\beta \in \mathbb{R}} \operatorname{ker} \pi_{\beta}=\bigcap_{\beta \in[0,1]} \operatorname{ker} \pi_{\beta}=\mathcal{J},
$$

as desired.

The spectrum of a 1D almost Mathieu operator may be approximated by considering its compressions to finite dimensional subspaces of the canonical basis of $\ell^{2}(\mathbb{Z})$ (cf. [Arv]), and this can be done in an effective way as the matrices one has to work with are tridiagonal selfadjoint matrices (as done in the computer program pending with [Arv]). Using [Bed2], the spectrum of a 2D almost Mathieu operator may also be approximated by the same method, but now it seems hard to do this in an effective way as the matrices are not tridiagonal in this case and one quickly runs into numerical problems.

If one of the components of $\Theta$ is an integer, one may introduce some generalized 1D almost Mathieu operators, as we do below, to which one should be able to adapt Arveson's program.

5.11 We assume for simplicity that $\Theta=\left(0, \theta_{2}, \theta_{3}\right)$ throughout this subsection.

When $\theta, \beta \in \mathbb{R}$, we recall from Section 2 that $M_{\theta, \beta}$ denotes the multiplication operator acting on $\ell^{2}(\mathbb{Z})$ associated to the function

$$
m \longrightarrow 2 \cos (2 \pi(m \theta+\beta)) .
$$

We also recall that $\Delta$ denotes the Laplacian on $\ell^{2}(\mathbb{Z})$.

Let now $\mathbf{c} \in \mathbb{R}^{3}, \beta_{2}, \beta_{3} \in \mathbb{R}$. We define the generalized $1 D$ almost Mathieu operator $h_{\Theta, \mathbf{c}, \beta_{2}, \beta_{3}}$ as the operator acting on $\ell^{2}(\mathbb{Z})$ given by

$$
h_{\Theta, \mathbf{c}, \beta_{2}, \beta_{3}}=c_{1} \Delta+c_{2} M_{\theta_{3}, \beta_{3}}+c_{3} M_{\theta_{2}, \beta_{2}} .
$$

We first etablish the connection with $\Delta_{\Theta, \mathbf{c}}$, which motivates the somewhat surprising ordering of indices in this definition. Let $z_{2}, z_{3} \in \mathbb{T}$. We define unitary 
operators $U, V_{z_{2}}$ and $W_{z_{3}}$ acting on $\ell^{2}(\mathbb{Z})$ by

$$
\begin{aligned}
U(\xi)(m) & =\xi(m-1), \\
V_{z_{2}} & =\text { multiplication by the function } m \longrightarrow z_{2} e^{-i 2 \pi m \theta_{3}}, \\
W_{z_{3}} & =\text { multiplication by the function } m \longrightarrow z_{3} e^{i 2 \pi m \theta_{2}} .
\end{aligned}
$$

Then one checks that $U, V_{z_{2}}$ and $W_{z_{3}}$ satisfy the following relations

$$
\begin{cases}U V_{z_{2}} & =e^{i 2 \pi \theta_{3}} V_{z_{2}} U, \\ V_{z_{2}} W_{z_{3}} & =W_{z_{3}} V_{z_{2}}, \\ W_{z_{3}} U & =e^{i 2 \pi \theta_{2}} U W_{z_{3}} .\end{cases}
$$

Using the universal property of $\mathcal{A}_{\Theta}$, there exists a homomorphism $\pi_{z_{2}, z_{3}}$ from $\mathcal{A}_{\Theta}$ onto $C^{*}\left(U, V_{z_{2}}, W_{z_{3}}\right)$ such that

$$
\pi_{z_{2}, z_{3}}\left(U_{1}\right)=U, \pi_{z_{2}, z_{3}}\left(U_{2}\right)=V_{z_{2}}, \pi_{z_{2}, z_{3}}\left(U_{3}\right)=W_{z_{3}} .
$$

Now, if we write $z_{2}=e^{-i 2 \pi \beta_{3}}, z_{3}=e^{i 2 \pi \beta_{2}}$, for some $\beta_{2}, \beta_{3} \in \mathbb{R}$, then a short computation gives

$$
\pi_{z_{2}, z_{3}}\left(\Delta_{\Theta, \mathbf{c}}\right)=h_{\Theta, \mathbf{c}, \beta_{2}, \beta_{3}} .
$$

Further, as to be expected, we get again an analogous result as in the $2 \mathrm{D}$ case. Note that with our standing assumption, $\Theta$ is degenerate when $1, \theta_{2}$ and $\theta_{3}$ are rationally dependent, and nondegenerate otherwise.

Proposition. We have

$$
S(\Theta, \mathbf{c})= \begin{cases}S p\left(h_{\Theta, \mathbf{c}, \beta_{2}, \beta_{3}}\right) & \text { if } \Theta \text { is nondegenerate, } \\ \bigcup_{\beta_{2}, \beta_{3} \in[0,1]} S p\left(h_{\Theta, \mathbf{c}, \beta_{2}, \beta_{3}}\right) & \text { if } \Theta \text { is degenerate. }\end{cases}
$$

Proof. The proof follows the same lines as the proof of Proposition 5.10, so let us be somewhat more sketchy. The nondegenerate case should be clear. In the degenerate case, it is enough to show that the family $\left\{\pi_{z_{2}, z_{3}}\right\}_{z_{2}, z_{3} \in \mathbb{T}}$ is separating, and this is done by showing that $\mathcal{K}$ is invariant under the action $\alpha$ on $\mathcal{A}_{\Theta}$, where

$$
\mathcal{K}:=\bigcap_{z_{2}, z_{3} \in \mathbb{T}} \operatorname{ker} \pi_{z_{2}, z_{3}} .
$$

For $z_{1} \in \mathbb{T}$, denote by $U_{z_{1}}$ the unitary operator acting on $\ell^{2}(\mathbb{Z})$ given as the multiplication operator associated with the function

$$
m \longrightarrow\left(z_{1}\right)^{m} .
$$

Then, for $z_{1}, z_{2}, z_{3} \in \mathbb{T}$, define a representation $\pi_{z_{1}, z_{2}, z_{3}}$ of $\mathcal{A}_{\Theta}$ on $\ell^{2}(\mathbb{Z})$ by

$$
\pi_{z_{1}, z_{2}, z_{3}}=\left(A d U_{z_{1}}\right) \circ \pi_{z_{2}, z_{3}} .
$$


Let $A \in \mathcal{K}, \gamma \in \mathbb{T}^{3}$. As

$$
\pi_{z_{1}, z_{2}, z_{3}} \circ \alpha_{\gamma}=\pi_{z_{1} \gamma_{1}, z_{2} \gamma_{2}, z_{3} \gamma_{3}},
$$

we get

$$
\begin{aligned}
\pi_{z_{2}, z_{3}}\left(\alpha_{\gamma}(A)\right) & =\pi_{\gamma_{1}, z_{2} \gamma_{2}, z_{3} \gamma_{3}}(A) \\
& =\left(A d U_{\gamma_{1}}\right)\left(\pi_{z_{2} \gamma_{2}, z_{3} \gamma_{3}}(A)\right) \\
& =0,
\end{aligned}
$$

for all $z_{2}, z_{3} \in \mathbb{T}$, hence $\alpha_{\gamma}(A) \in \mathcal{K}$ as desired.

This result is interesting in several aspects. First, is clear that generalized 1D almost Mathieu operators are 1D discrete almost periodic Schrödinger operators (sometimes called almost periodic Jacobi matrices, cf. [Sim, CFKS]). Quite a lot is known about this class of operators and it has been observed that the spectrum of such an operator has a tendency to be a Cantor set. Hence, the above result supports the idea that the spectrum of a 3D discrete magnetic Laplacians could always (or at least generically) be a Cantor set in the nondegenerate case.

Secondly, it is not difficult to see that one may use the method of Arveson ([Arv, Bed2]) to approximate spectra of generalized 1D almost Mathieu operators, and this may be done effectively as the matrices to be diagonalized are now tridiagonal, cf. our comment at the end of 5.10. Hence the above result may be used as a basis for approximating spectra of 3D discrete magnetic Laplacians (for the class of $\Theta$ considered in this section), in a different way than the one alluded to in 5.7. Again, we hope to come back to these numerical considerations in a later work.

Finally, it opens up the possibility that one may be able to adapt some of the arguments used in the study of $2 \mathrm{D}$ discrete magnetic Laplacians to the 3D case considered here. For example, we have checked that one may adapt the proof of Theorem 5.3 in [CEY], to show that the map $\Theta \longrightarrow S(\Theta$, c) is Hausdorffcontinuous when restricted to the set of $\Theta$ with $\theta_{1}=0$, cf. our comment in 5.8. Note that the tridiagonality of generalized $1 \mathrm{D}$ almost Mathieu operators is important for carrying out the argument.

5.12 We have written this paper as an introductory text and there is clearly a lot of work left to be done. Besides the questions mentionned so far, one may ask e. g. wether anything can be said about the Lebesgue measure of $S(\Theta, \mathbf{c})$, or about its Hausdorff dimension. Another question is wether it is possible to use semiclassical analysis ([Bel4, HS, Sjo]) in the study of $S(\Theta, \mathbf{c})$. We hope this article will succeed in drawing attention to this fascinating subject, which we think deserves to be investigated more thoroughly.

Acknowledgements. We are indebted to J. Bellissard, G.A. Elliott and the participants of the $\mathrm{C}^{*}$-seminar at the University of Oslo for some helpful conversations at various stages of this work. We also take the opportunity to thank S. Gaure for introducing us to $\mathrm{HAT}_{\mathrm{E}} \mathrm{X}$. 


\section{References}

[AP] J. Anderson, W. Paschke : The rotation algebra. Houston J. Math. 15 (1989), 1-26.

[Arv] W. Arveson : $C^{*}$-algebras and numerical linerar algebra. J. Funct. Anal. 122 (1994), 333-360.

[AS] J. Avron, B. Simon : Almost periodic Schrödinger operators II. The integrated density of states. Duke Math. J. 50 (1983), 369-391.

[Bac] N. B. Backhouse : Projective representations of space groups, II: Factor systems. Quart. J. Math. Oxford 21 (1970), 223-242.

[BB] N. B. Backhouse, C. J. Bradley : Projective representations of space groups, I: Translation groups. Quart. J. Math. Oxford 21 (1970), 203222.

[Bed1] E. Bédos : Simple $C^{*}$-crossed products with a unique trace. Ergod. Th. and Dyn. Sys. 16 (1996), 415-429.

[Bed2] E. Bédos : On Fölner nets, Szegö's theorem and other eigenvalue distribution theorems. Expo. Math. 15 (1997), 193-228.

[Bed3] E. Bédos : Work in preparation.

[BVZ] C. Béguin, A. Valette, A. Zuk: On the spectrum of a random walk on the discrete Heisenberg group. J. Geom. and Phys. 21 (1997), 337-356.

[Bel1] J. Bellissard : K-theory of $C^{*}$-algebras in solid state physics. Springer Lect. Notes in Phys. 257 (1986), 99-156.

[Bel2] J. Bellissard : $C^{*}$-algebras in solid state physics $-2 D$ electrons in a uniform magnetic field In D. Evans, M. Takesaki (eds) : Operator algebras and applications, vol. 2. Cambrige University Press, 1988, 49-76.

[Bel3] J. Bellissard : Almost periodicity in solid state physics and $C^{*}$ algebras. The Harald Bohr centenary (C. Berg, B. Fuglede, eds. ). Royal Danish Academy of Sciences, MfM 42:3 (1989), 35-75.

[Bel4] J. Bellissard : Le papillon de Hofstadter (d'après B. Helffer et J. Sjöstrand). Sém. Bourbaki, Exposé 745 (1991).

[Bel5] J. Bellissard : Gap labelling theorems for Schrödinger operators. In M. Waldschmidt (eds) : From number theory to physics. Springer Verlag, 1992, 538-630.

[Bel6] J. Bellissard : Lipshitz continuity of gap boundaries for Hofstadter-like spectra. Commun. Math. Phys. 160 (1994), 599-613. 
[BES] J. Bellissard, A. van Elst, H. Schulz-Bades : The non-commutative geometry of the quantum Hall effect. J. Math. Phys. 35 (1994), 53735451.

[BS] J. Bellissard, B. Simon : Cantor spectrum for the almost Mathieu equation. J. Funct. Anal. 48 (1982), 408-419.

[Bla] B. Blackadar : K-theory for operator algebras M. S. R. I. Publ. 5, Springer Verlag, 1986.

[BKR] B. Blackadar, A. Kumjian, M. Rørdam : Approximately central matrix units and the structure of noncommutative tori. K-Theory 6(1992), 267-284.

[Boc] F. P. Boca: The structure of higher-dimensional noncommutative tori and metric diophantine approximation. J. Reine Angew. Math. 492 (1997), 179-219.

[Bre] B. Brenken : A classification of some noncommutative tori. Rocky Mount. J. Math. 20 (1990), 389-397.

[BCEN] B. Brenken, J. Cuntz, G. A. Elliott, R. Nest : On the classification of noncommutative tori, III. Comtemp. Math. Amer. Math. Soc. 60 (1986), 503-526.

[Bro] E. Brown : Bloch electrons in a uniform magnetic field. Phys. Rev. 133 (1964), 1038-1044.

[CEY] M. D. Choi, G. A. Elliott, N. Yui : Gauss polynomials and the rotation algebra. Invent. Math. 99 (1990), 225-246.

[Con1] A. Connes : Classification of injective factors. Ann. Math. 104 (1976), 73-115.

[Con2] A. Connes : Noncommutative geometry. Academic Press, 1994.

[CEGJ] J. Cuntz, G. A. Elliott, F. M. Goodman, P. E. T. Jorgensen : On the classification of noncommutative tori, II. C. R. Math. Rep. Acad. Sci. Canada 7 (1985), 189-194.

[CFKS] H. L. Cycon, R. G. Froese, W. Kirsch, B. Simon : Schrödinger operators with applications to quantum mechanics and global geometry. Springer Verlag, 1987.

[DS] F. Delyon, B. Souillard : Remark on the continuity of the density of states of ergodic finite differences operators. Commun. Math. Phys. 94 (1984), 289-291.

[Ell1] G. A. Elliott : Gaps in the spectrum of an almost periodic Schrödinger opertor. C. R. Math. Rep. Acad. Sci. Canada 4 (1982), 255-259. 
[El12] G. A. Elliott : On the $K$-theory of the $C^{*}$-algebra generated by the projective representation of a discrete abelian group. In : Operator algebras and group representations, vol. 1, Pitman, 1984, 157-184.

[El13] G. A. Elliott : On the classification of $C^{*}$-algebras of real rank zero. J. Reine Angew. Math. 443 (1993), 179-219.

[EE] G. A. Elliott, D. E. Evans : The structure of the irrational rotation $C^{*}$-algebra. Ann. Math. 138 (1993), 477-501.

[EL1] G. A. Elliott, Q. Lin : Cut down method in the inductive limit decomposition of noncommutative tori. J. Lond. Math. Soc. 54 (1996), 121-134.

[EL2] G. A. Elliott, Q. Lin : Cut down method in the inductive limit decomposition of noncommutative tori. Part II. Fields Ins. Commun. 13 (1997), 91-123.

[ENN] G. A. Elliott, T. Natsume, R. Nest : The Heisenberg group and Ktheory. K-theory 7 (1993), 409-428.

[Fil] P. A. Fillmore : A user's guide to operator algebras. A WileyInterscience publication, John Wiley and Sons, New-York , 1996.

[HR] U. Haagerup, M. Rørdam : Perturbations of the rotation $C^{*}$-algebras and of the Heisenberg commutation relation. Duke Math. J. 77 (1995), $627-656$.

[HS] B. Helffer, J. Sjöstrand: On diamagnetism and de Haas-van Alphen effect. Ann. Inst. Henri Poincaré Phys. Th. 52 (1990), 303-375.

[Hun] W. Hunziker : Schrödinger operators with electric or magnetic fields. Springer Lect. Notes in Phys. 116 (1979), 25-44.

[Lan] L. D. Landau : Diamagnetismus der Metalle. Z. f. Phys. 64 (1930), 629-637.

[LL] L. D. Landau, E. M. Lifschitz : Quantum mechanics - Non-relativistic theory. Pergamon, New-York (2nd ed), 1965.

[LiLo] E. H. Lieb, M. Loss : Fluxes, Laplacians and Kasteleyn's theorem. Duke Math. J. 71 (1993), 337-363.

[Las] Y. Last : Zero measure spectrum for the almost Mathieu operator. Commun. Math. Phys. 164 (1994), 421-432.

[Lin] Q. Lin : Cut down method in the inductive limit decomposition of noncommutative tori. Part III. Commun. Math. Phys. 179 (1996), $555-575$. 
[NT] K. Egede Nielsen, K. Thomsen : Limits of circle algebras. Expo. Math. 14 (1996), 17-56.

[OPT] D. Olesen, G. K. Pedersen, M. Takesaki : Ergodic actions of compact abelian groups. J. Operator Theory 3 (1980), 237-269.

[Pac] J. Packer : Twistwd group $C^{*}$-algebras corresponding to nilpotent discrete groups. Math. Scand. 64 (1989), 109-122.

[PV] M. V. Pimsner, D. Voiculescu : Exact sequences for K-groups and Extgroups of certain $C^{*}$-crossed products. J. Operator Theory 4 (1980), 93-118.

[RS] M. Reed, B. Simon : Methods of modern mathematical physics, I-IV. Academic Press, 1974-1979.

[Rie1] M. A. Rieffel : $C^{*}$-algebras associated with irrational rotations. Pac. J. Math. 93 (1981), 415-429.

[Rie2] M. A. Rieffel : Projective modules over higher-dimensional noncommutative tori. Can. J. Math. 40 (1988), 257-338.

[Rie3] M. A. Rieffel : Continuous fields of $C^{*}$-algebras coming from group cocycles and actions. Math. Ann. 283 (1989), 631-643.

[Rie4] M. A. Rieffel : Noncommutative tori - a case study of noncommutative differentiable manifolds. Contemp. Math. 105 (1990), 191-211.

[Shu] M. A. Shubin : Discrete Magnetic Laplacians. Commun. Math. Phys. 164 (1994), 259-275.

[Sim] B. Simon : Almost periodic Schrödinger operators: a review. Adv. Appl. Math. 3 (1982), 463-490.

[Sjo] J. Sjöstrand: Microlocal analysis for the periodic magnetic Schrödinger equation and related questions. Springer Lect. Notes in Math. 1495 (1991), 237-332.

[Sla] J. Slawny : On factor representations and the $C^{*}$-algebra of canonical commutation relations. Commun. Math. Phys. 24 (1972), 151-170.

[Sun] T. Sunada : A discrete analogue of periodic magnetic Schrödinger operators. Contemp. Math. 173 (1994), 283-299.

[Wat] Y. Watatani: Toral automorphisms of irrational rotation algebras. Math. Jap. 26 (1981), 479-484.

[Yin] H.-S. Yin : A simple proof of the classification of rational rotation $C^{*}$-algebras. Proc. Amer. Math. Soc. 98 (1986), 469-470.

[Zak] J. Zak : Magnetic translation group. Phys. Rev. 134 (1964), 16021606. 
[ZM] G. Zeller-Meier : Produits croisés d'une $C^{*}$-algèbre par un groupe d'automorphismes. J. Math. Pures Appl. 47 (1968), 101-239.

Institute of Mathematics, University of Oslo, PB 1053 Blindern, 0316 Oslo, Norway.

E-mail:bedos@math.uio.no 